\title{
Decentralized Coordinated Precoding for Dense TDD Small Cell Networks
}

\author{
Sandra Lagen, Adrian Agustin, Member, IEEE, and Josep Vidal, Member, IEEE
}

\begin{abstract}
Cellular networks need the densification of Small eNBs (SeNBs) to face the tremendous data traffic demand growth, implying an interference increase and making transmit coordination a key enabler. This article proposes a decentralized coordinated precoding (D-CoP) for downlink (DL) weighted sumrate maximization in dense MIMO TDD small cell networks (SCNs). Each SeNB designs its own precoding matrices based on channel state information (CSI) of the served users and knowledge of the interference-cost matrix that allows managing interference towards unintended users. A protocol is proposed to acquire the interference-cost matrix by processing the uplink (UL) received signal provided that: $i$ ) channel reciprocity can be assumed and $i$ ) all users participating in DL can transmit in UL with an adequate transmit filter. In contrast to existing transmit coordination techniques, D-CoP is fully scalable, avoids estimation of the interfering channels, and does not require information exchange between SeNBs. In case all parameters are perfectly acquired, an iterative algorithm is presented with demonstrated monotonic convergence when all SeNBs update its transmit precoders simultaneously. Further, the problem is reformulated in order to derive a robust $\mathrm{D}$-CoP under imperfect CSI conditions. Finally, simulations in 3GPP LTE-Advanced SCNs show significant user packet throughput gains, without increasing the complexity associated to transmit coordination. Robustness to imperfect CSI and non-ideal channel reciprocity is shown through simulations.
\end{abstract}

Index Terms-multi-cell multi-user MIMO, decentralized coordinated precoding design, interference management, dense TDD small cell networks.

\section{INTRODUCTION}

The envisioned rapid and exponential increase of the wireless data traffic demand in the next years imposes rethinking current wireless cellular networks. In this regard, heterogeneous cellular networks (HCNs) are a promising solution to increase the capacity of future cellular systems, as LTEAdvanced (LTE-A) [2], through the densification of the network and the re-use of the spectrum [3]. HCNs consist of a multi-tier deployment of macrocells and small cells (encompassing picocells and femtocells), which are being extensively studied in academia, industry, and standardization bodies such as 3GPP [4]. While macrocells are covered by Macro evolved

Manuscript received November 2, 2014; revised February 24, 2015.

S. Lagen, A. Agustin, and J. Vidal are with the department of Signal Theory and Communications, Universitat Politècnica de Catalunya, Barcelona, Spain. e-mail: \{sandra.lagen, adrian.agustin, josep.vidal\}@upc.edu

This work has been supported by projects TROPIC FP7 ICT-2011-8318784 (European Commission), FPU12/00828 grant (Ministerio de Educación, Cultura y Deporte, Spanish Government), DISNET TEC2013-41315-R (Ministerio de Economía y Competitividad, Spanish Government and ERDF funds), and 2014SGR-60 (Catalan Government).

Part of this work has been presented in the 2013 IEEE Global Communications Conference (GLOBECOM) [1].
Node Bs (MeNBs), which are intended to provide general coverage and service to high mobility User Equipments (UEs), small cells are served by Small evolved Node Bs (SeNBs), which dispose of a lower transmit power, cover smaller areas, and are intended to serve low mobility UEs so as to avoid frequent handovers. This allows obtaining a reliable estimation of the propagation channel from the UE towards the serving SeNB due to the long channel coherence time [2].

An interesting type of deployment contemplated in 3GPP LTE-A networks is the non-co-channel HCNs deployment, where MeNBs and SeNBs use different carrier frequencies. This way, MeNBs may keep on working as usual and traffic can be offloaded to the new underlying small cell network (SCN) [5]. Although cross-tier interference can be eliminated, the co-tier interference between SeNBs becomes a major problem that can significantly reduce the system throughput if SeNBs are densely deployed in concentrated clusters that serve hot-spot areas with high user traffic demands [6]. One advantage of the $\mathrm{SCN}$ is that SeNBs are supposed to operate in time division duplex (TDD) mode so as to better match the uplink:downlink traffic asymmetry, which allows exploiting the reciprocity of uplink (UL) and downlink (DL) propagation channels to design advanced precoding techniques and hence improving the performance of the TDD SCN and reducing the channel feedback signaling needed in the frequency division duplex (FDD) mode [7].

The dense TDD SCN can be modeled as a multi-cell multi-user multiple-input multiple-output (MIMO) system, a generic model for multi-user cellular communication systems where multiple SeNBs, each equipped with multiple antennas, wish to simultaneously send independent messages to its served UEs while generating interference to unintended UEs. Unfortunately, the optimal transmit/receive strategy with linear filters that maximizes the weighted sum-rate (WSR) of the system is not known. From an optimization theory perspective, the maximum WSR problem is non-convex and NP-hard even in the single-antenna case [8], but several approaches that reach a local optima have been proposed in the literature. Some of them are centralized methods, whose main drawback is the added cost of a central unit required to collect the channel state information (CSI) of all UEs, and the scalability of the solution. This has sparked a great interest in developing decentralized approaches that work iteratively.

Iterative methods in [9][10] (and references therein) are based on the concept of the interference-cost, in which each SeNB maximizes its own utility function minus the interference-cost (that reflects the interference created towards unintended UEs). Methods are available to ensure the con- 
vergence of these algorithms by slightly reformulating the problem (see for example [10]), nevertheless, all channel matrices (i.e. direct and interfering channel matrices) have to be estimated and reported along with the generated interferencecost. An alternative approach is presented in [11] for WSR maximization that relies on a iterative minimization of the weighted sum of mean-square errors (WMSE), as was initially introduced in [12], where the solution is achieved by iteratively updating transmit filters at SeNBs and receive filters and weighting matrices at UEs. Although monotonic convergence is demonstrated, its decentralized implementation requires again estimation of all channel matrices, and the existence of feedback links from each UE towards all SeNBs is needed to report the updated receive filters and weighting matrices (see [11]). Therefore, the main drawbacks of existing approaches to solve the maximization of the WSR in the literature ([9]-[11]) are: $i$ ) the estimation of all channel matrices (as well as the associated computational cost and required network planning for channel estimation), ii) the impact of channel estimation errors of the interfering channel matrices that are estimated with a low signal-to-noise ratio (SNR), and iii) the use of nonideal backhaul and/or feedback links to exchange information among different terminals (SeNBs/UEs). All has a detrimental effect on the overall potential performance gains of transmit coordination techniques.

In the present work we exploit the fact that propagation channel reciprocity and a reliable estimation of the direct channels are available in TDD SCNs in order to propose a decentralized, scalable, and coordinated interference management procedure for maximizing the WSR in DL with linear transmit/receive filters. The WMSE formulation is used to face the maximization of the WSR, but different from [11] we decentralize the minimum WMSE problem by following the interference-cost concept such that a different transmit filters design at SeNBs is derived. Each SeNB designs its own transmit filters (precoders) based on the knowledge of the direct channel matrix towards the served UEs, the covariance matrix of the DL inter-cell interference plus noise that can be acquired from a reported parameter from the served UEs, and the interference-cost matrix that allows managing interference towards unintended users. We propose that each SeNB acquires the interference-cost matrix over-the-air by using the UL received signal, provided that all UEs currently participating in DL can transmit simultaneously a specific pilot sequence in UL (as is shown in Fig. 1). By doing so, and in contrast to previous works, the estimation of the interfering channel matrices is not needed, hence significantly reducing its associated complexity in terms of computational cost and network planning for pilot signals, and also reducing the performance degradation due to imperfect estimation. Furthermore, information exchange among SeNBs is not required, which alleviates the impact of non-ideal backhaul links, and the number of feedback links and amount of information from UEs to SeNBs are significantly reduced as compared to [11].

The contributions of this work are:

- A decentralized coordinated precoding (D-CoP) for DL WSR maximization is proposed, in which each SeNB solves its own subproblem to design transmit filters.

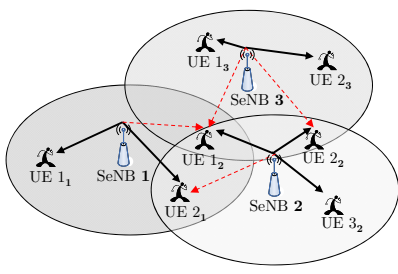

(a) DL desired signals (solid) and interfering signals (dashed)



(b) UL useful signals to acquire the interference-cost (solid)
Fig. 1: Acquisition of the interference-cost matrix from the UL transmission for DL transmit coordination in dense TDD Small Cell Networks.

- The subproblem is reformulated to tackle imperfect CSI conditions and a robust $\mathrm{D}-\mathrm{CoP}$ is derived to overcome estimation errors of the direct channel matrices at SeNBs.

- A new protocol is proposed to estimate the interferencecost matrix at each SeNB over-the-air from an UL pilotbased transmission.

- An iterative algorithm for D-CoP is presented, subsuming the acquisition of the required parameters at SeNBs and the simultaneous per-SeNB transmit filters design.

- Monotonic convergence of the algorithm for D-CoP is demonstrated if all parameters are perfectly acquired.

- The proposed D-CoP is evaluated in a 3GPP LTE-A small cell scenario [6], showing large DL performance gains even when only 1 iteration is implemented.

Although the proposed D-CoP is applied to TDD SCNs in this work, it can be used to control interference in the general multi-cell multi-user MIMO TDD systems.

Organization: The rest of the paper is organized as follows. In Section II the system model is presented, including the signal model for channel estimation, DL and UL transmissions. In Section III the maximum WSR problem is formulated. In Section IV the proposed D-CoP is derived assuming either perfect CSI or imperfect CSI conditions for the direct links, and how to acquire the required parameters for decentralized transmit filters design at SeNB is detailed. The iterative algorithm for $\mathrm{D}-\mathrm{CoP}$ is presented in Section $\mathrm{V}$, including convergence and complexity considerations and analyzing practical implementation issues. In Section VI the proposed procedure is evaluated using a 3 GPP LTE-A SCN [6]. Finally, concluding remarks are included in Section VII.

Notation: In this paper, italic letters, boldface lower-case and upper-case letters denote scalars, vectors and matrices, respectively. For given scalars $a$ and $b, \min (a, b)$ denotes the minimum between $a$ and $b, \log _{2}(a)$ refers to the base2 logarithm, $\ln (a)$ to the natural logarithm, and $\operatorname{Pr}(a<b)$ describes the probability of $a$ being smaller than $b$. For a given matrix $\mathbf{A}$, its transpose matrix is denoted as $\mathbf{A}^{T}$, its conjugate matrix as $\mathbf{A}^{*}$, its transpose conjugate matrix as $\mathbf{A}^{H}$, and the matrix inverse as $\mathbf{A}^{-1} \cdot|\mathbf{A}|$ operator refers to the determinant of $\mathbf{A}, \operatorname{Tr}(\mathbf{A})$ to the trace, $\mathbb{E}_{\mathbf{x}}[\mathbf{A}]$ to the expectation operator with respect to variable $\mathbf{x}$, and $\mathbb{E}_{\mathbf{x}}[\mathbf{A} \mid \mathbf{B}]$ to the expectation operator with respect to variable $\mathbf{x}$ for a known matrix $\mathbf{B}$. Matrix I refers to the identity matrix. $\mathbb{C}^{m \times n}$ denotes an $m$ by $n$ dimensional complex space. The complex normal distribution is represented by $\mathcal{C N}(.,$.$) and the real truncated normal distribution in the$ interval $[a, b]$ is denoted by $\mathcal{T} \mathcal{N}(., ., a, b)$. 


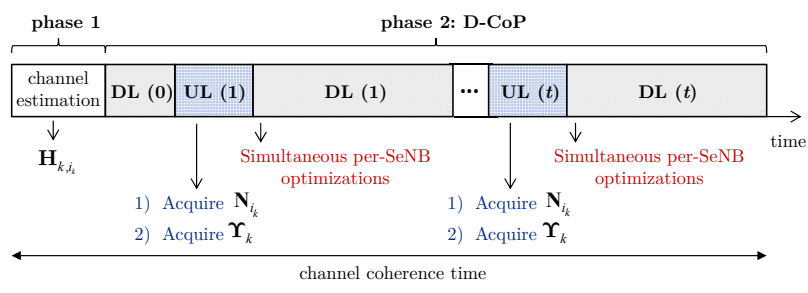

Fig. 2: Algorithm for D-CoP in TDD Small Cell Networks.

\section{SYSTEM MODEL}

Consider a TDD SCN composed $K$ SeNBs, where the $k$-th SeNB $(k=1, \ldots, K)$ is equipped with $M_{k}$ transmit antennas and serves $I_{k}$ UEs. Let us define $i_{k}$ to be the $i$-th UE served by the $k$-th $\mathrm{SeNB}$ and $N_{i_{k}}$ the receive antenna elements at the $i_{k}$-th UE $\left(i=1, \ldots, I_{k}\right)$. The total number of UEs is: $I=\sum_{k=1}^{K} I_{k}$. An example is shown in Fig. 1 for $K=3$ and $I=7\left(I_{1}=2, I_{2}=3, I_{3}=2\right)$. Through the paper, we use $i$, $j$ to indicate the UE index and $k, l$ to indicate the SeNB index. All SeNBs present in the cellular network transmit information simultaneously to its served UEs, such that severe interference is created. Our focus is on DL interference management.

Low mobility UEs are assumed to be connected to the synchronized TDD SCN. During the channel coherence time, the communication is split into 2 phases (as shown in Fig. 2):

- Phase 1 (channel estimation): Phase 1 is devoted to acquire knowledge at each $k$-th SeNB of the direct channel matrix towards its served $i_{k}$-th UE, $\forall i$, which is denoted by $\mathbf{H}_{k, i_{k}} \in \mathbb{C}^{N_{i_{k}} \times M_{k}}$ and contains the complexvalued channel gains of the different antenna-pairs.

- Phase 2 (D-CoP algorithm): In phase 2, UL and DL transmissions are duplexed. The proposed algorithm iterates between UL and DL: in UL transmissions parameters like the DL inter-cell interference plus noise covariance matrix of the served $i_{k}$-th UE $\left(\mathbf{N}_{i_{k}}, \forall i\right)$ and the interference-cost matrix for the $k$-th $\operatorname{SeNB}\left(\boldsymbol{\Upsilon}_{k}\right)$ are acquired at each $k$-th SeNB, which are used for decentralized transmit filters design in the subsequent DL transmission. See details in Section IV.

In TDD systems, channel estimation can be done either at the SeNB or at the UE. In the later case, a feedback link is needed to report the channel matrix to the serving SeNB. For the ease of exposition, we consider that the direct channel matrix is estimated at the SeNB from an UL pilot-based transmission assuming perfect UL-DL propagation channel reciprocity (i.e. the channel matrix in UL $\overleftarrow{\mathbf{H}}_{k, i_{k}} \in \mathbb{C}^{M_{k} \times N_{i_{k}}}$ is given by the transpose of the channel matrix in DL: $\left.\overleftarrow{\mathbf{H}}_{k, i_{k}}=\mathbf{H}_{k, i_{k}}^{T}, \forall k, \forall i\right)$. See details in subsection II-A.

Different from previous works ([9][11]), the proposed DCoP scheme does not need to know the interfering channel matrices (i.e. $\mathbf{H}_{k, j_{l}} \in \mathbb{C}^{N_{j_{l}} \times M_{k}}$, between the $k$-th SeNB and the $j_{l}$-th UE served by $l$-th SeNB, $\forall l \neq k$ ) either at SeNBs or at UEs. Consequently, we avoid: $i$ ) the computational cost associated to channel estimation, ii) the network planning required for pilot signals, and iii) the performance loss due to imperfect estimation of the interfering channel matrices that are estimated with a low SNR.

\section{A. Signal model for channel estimation (phase 1)}

For channel estimation at SeNBs we use a well-established orthogonal training-based scheme [13] such that interference between neighbor UEs for CSI acquisition is avoided. Hence, the signal received by the $k$-th SeNB at the $M_{k}$ receive antennas during $T \geq N_{i_{k}}$ channel uses $\mathbf{S}_{k} \in \mathbb{C}^{M_{k} \times T}$ is:

$$
\mathbf{S}_{k}=\mathbf{H}_{k, i_{k}}^{T} \mathbf{P}_{i_{k}}+\mathbf{V}_{k}
$$

where $\mathbf{H}_{k, i_{k}} \in \mathbb{C}^{N_{i_{k}} \times M_{k}}$ is the real channel matrix, $\mathbf{P}_{i_{k}} \in \mathbb{C}^{N_{i_{k}} \times T}$ is the known matrix of training sequences for the $i_{k}$-th UE, and $\mathbf{V}_{k} \in \mathbb{C}^{M_{k} \times T}$ is the collection of channel noise vectors, composed of Gaussian independent and identically distributed (i.i.d.) components with distribution $\mathcal{C N}\left(0, \sigma_{v}^{2}\right)$. Let us denote $P_{T}$ to the total power for channel estimation, i.e. $P_{T}=\operatorname{Tr}\left(\mathbf{P}_{i_{k}} \mathbf{P}_{i_{k}}^{H}\right)$. Channel matrix $\mathbf{H}_{k, i_{k}}$ is modeled by Gaussian i.i.d. components with distribution $\mathcal{C N}\left(0, \delta_{k, i_{k}}\right)$, where $\delta_{k, i_{k}}$ refers to the gain introduced by pathloss and shadowing between the $k$-th SeNB and the $i_{k}$-th UE.

The channel estimation model is described by:

$$
\mathbf{H}_{k, i_{k}}=\hat{\mathbf{H}}_{k, i_{k}}+\tilde{\mathbf{H}}_{k, i_{k}},
$$

where $\hat{\mathbf{H}}_{k, i_{k}}$ is the estimated channel matrix and $\tilde{\mathbf{H}}_{k, i_{k}}$ is the channel estimation error matrix. We assume that the channel matrix is estimated at SeNBs by applying a linear minimum mean square error (MMSE) estimator [14], i.e.

$$
\hat{\mathbf{H}}_{k, i_{k}}^{T}=\mathbb{E}_{\mathbf{H} \mid \mathbf{S}}\left[\mathbf{H}_{k, i_{k}}^{T} \mid \mathbf{S}_{k}\right]=\mathbf{S}_{k} \mathbf{P}_{i_{k}}^{H}\left(\mathbf{P}_{i_{k}} \mathbf{P}_{i_{k}}^{H}+\frac{\sigma_{v}^{2}}{\delta_{k, i_{k}}} \mathbf{I}\right)^{-1} .
$$

Therefore, assuming mutually white and orthogonal training sequences (i.e. $\mathbf{P}_{i_{k}}$ is a unitary matrix scaled by $\sqrt{P_{T} / N_{i_{k}}}$ ), the components of $\tilde{\mathbf{H}}_{k, i_{k}}$ in (2) turn out to be Gaussian and mutually uncorrelated with a variance for each channel estimation error component given by [14]:

$$
\bar{J}_{k, i_{k}}=\frac{\operatorname{Tr}\left(\mathbb{E}_{\tilde{\mathbf{H}}}\left[\tilde{\mathbf{H}}_{k, i_{k}}^{T} \tilde{\mathbf{H}}_{k, i_{k}}^{*}\right]\right)}{M_{k} N_{i_{k}}}=\left(\frac{1}{\delta_{k, i_{k}}}+\frac{P_{T}}{N_{i_{k}} \sigma_{v}^{2}}\right)^{-1} .
$$

For simplicity, it is assumed that noise power in the channel estimation phase is equal for all links, such that the different variances in the estimation errors in (4) come from the pathloss and shadowing variations of the different links.

\section{B. Signal model for downlink (phase 2)}

The signal transmitted by the $k$-th SeNB in DL is given by:

$$
\mathbf{x}_{k}=\sum_{i=1}^{I_{k}} \mathbf{T}_{i_{k}} \mathbf{b}_{i_{k}}
$$

where $\mathbf{b}_{i_{k}} \in \mathbb{C}^{m_{i_{k}} \times 1}$ contains the unit power independent Gaussian symbols for the $i_{k}$-th UE (i.e. $\mathbf{b}_{i_{k}} \sim \mathcal{C N}(\mathbf{0}, \mathbf{I})$ ) and $\mathbf{T}_{i_{k}} \in \mathbb{C}^{M_{k} \times m_{i_{k}}}$ denotes its associated transmit filter (or precoder), being $m_{i_{k}} \leq \min \left(N_{i_{k}}, M_{k}\right)$ the number of streams. The total power spent at the $k$-th SeNB is:

$$
P_{k}=\sum_{i=1}^{I_{k}} \operatorname{Tr}\left(\mathbf{T}_{i_{k}} \mathbf{T}_{i_{k}}^{H}\right) .
$$

Assuming narrow-band transmissions, the equivalent base- 
band signal observed at the $i_{k}$-th UE is:

$$
\begin{aligned}
\mathbf{y}_{i_{k}}= & \mathbf{H}_{k, i_{k}} \mathbf{T}_{i_{k}} \mathbf{b}_{i_{k}} \\
& +\underbrace{\sum_{j=1, j \neq i}^{I_{k}} \mathbf{H}_{k, i_{k}} \mathbf{T}_{j_{k}} \mathbf{b}_{j_{k}}}_{\text {intra-cell interference }}+\underbrace{\sum_{l=1, l \neq k}^{K} \sum_{j=1}^{I_{l}} \mathbf{H}_{l, i_{k}} \mathbf{T}_{j_{l}} \mathbf{b}_{j_{l}}}_{\text {inter-cell interference }}+\mathbf{v}_{i_{k}},(7)
\end{aligned}
$$

where $\mathbf{H}_{l, i_{k}} \in \mathbb{C}^{N_{i_{k}} \times M_{l}}$ is the channel matrix between the transmitting antennas at the $l$-th SeNB and the receiving antennas at the $i_{k}$-th UE. The second term in (7) contains the intra-cell interference, the third term the inter-cell interference, and last term refers to the additive zero-mean white Gaussian noise with distribution $\mathbf{v}_{i_{k}} \sim \mathcal{C N}\left(\mathbf{0}, \sigma_{i_{k}}^{2} \mathbf{I}\right)$. Hence, under the independence assumption of $\left\{\mathbf{b}_{i_{k}}\right\}, \forall i, \forall k$, and $\mathbf{v}_{i_{k}}$, the covariance matrix of the received signal at the $i_{k}$-th $\mathrm{UE}$ is:

$$
\mathbf{C}_{\mathbf{y}_{i_{k}}}=\mathbb{E}_{\mathbf{b}, \mathbf{v}}\left[\mathbf{y}_{i_{k}} \mathbf{y}_{i_{k}}^{H}\right]=\sum_{j=1}^{I_{k}} \mathbf{H}_{k, i_{k}} \mathbf{T}_{j_{k}} \mathbf{T}_{j_{k}}^{H} \mathbf{H}_{k, i_{k}}^{H}+\mathbf{N}_{i_{k}},
$$

where $\mathbf{N}_{i_{k}}$ denotes the covariance matrix of the DL inter-cell interference plus noise at the $i_{k}$-th UE:

$$
\mathbf{N}_{i_{k}}=\sum_{l=1, l \neq k}^{K} \sum_{j=1}^{I_{l}} \mathbf{H}_{l, i_{k}} \mathbf{T}_{j_{l}} \mathbf{T}_{j_{l}}^{H} \mathbf{H}_{l, i_{k}}^{H}+\sigma_{i_{k}}^{2} \mathbf{I} .
$$

The symbols are estimated at the $i_{k}$-th UE assuming that interference is treated as noise and that a linear receive filter $\mathbf{R}_{i_{k}} \in \mathbb{C}^{N_{i_{k}} \times m_{i_{k}}}$ is applied at UE:

$$
\hat{\mathbf{b}}_{i_{k}}=\mathbf{R}_{i_{k}}^{H} \mathbf{y}_{i_{k}} \text {. }
$$

The mean square error (MSE) for the symbols transmitted towards the $i_{k}$-th UE can be expressed through the so-called MSE-matrix $\mathbf{E}_{i_{k}}=\mathbb{E}_{\mathbf{b}, \mathbf{v}}\left[\left(\hat{\mathbf{b}}_{i_{k}}-\mathbf{b}_{i_{k}}\right)\left(\hat{\mathbf{b}}_{i_{k}}-\mathbf{b}_{i_{k}}\right)^{H}\right]$, which can be expressed in terms of $\mathbf{R}_{i_{k}}$ in (10) and $\left\{\mathbf{T}_{i_{k}}\right\}$ in (5):

$$
\begin{aligned}
\mathbf{E}_{i_{k}}\left(\mathbf{R}_{i_{k}},\left\{\mathbf{T}_{i_{k}}\right\}\right)= & \mathbf{I}+\mathbf{R}_{i_{k}}^{H}\left(\sum_{j=1}^{I_{k}} \mathbf{H}_{k, i_{k}} \mathbf{T}_{j_{k}} \mathbf{T}_{j_{k}}^{H} \mathbf{H}_{k, i_{k}}^{H}+\mathbf{N}_{i_{k}}\right) \mathbf{R}_{i_{k}} \\
& -\mathbf{R}_{i_{k}}^{H} \mathbf{H}_{k, i_{k}} \mathbf{T}_{i_{k}}-\mathbf{T}_{i_{k}}^{H} \mathbf{H}_{k, i_{k}}^{H} \mathbf{R}_{i_{k}} .
\end{aligned}
$$

The achievable rate of the $i_{k}$-th UE is understood as the well-known "log-det" capacity formula:

$$
R_{i_{k}}\left(\left\{\mathbf{T}_{i_{k}}\right\}\right)=\log _{2}\left|\mathbf{I}+\mathbf{H}_{k, i_{k}} \mathbf{T}_{i_{k}} \mathbf{T}_{i_{k}}^{H} \mathbf{H}_{k, i_{k}}^{H} \mathbf{N}_{i_{k}}^{-1}\right| .
$$

In case of imperfect CSI of the direct channel matrix $\mathbf{H}_{k, i_{k}}$, the MSE-matrix in (11) has to be averaged with respect to the conditional probability density function of the channel estimation errors given the channel estimate [15]: $\overline{\mathbf{E}}_{i_{k}}=\mathbb{E}_{\mathbf{b}, \mathbf{v}, \tilde{\mathbf{H}} \mid \hat{\mathbf{H}}}\left[\left(\hat{\mathbf{b}}_{i_{k}}-\mathbf{b}_{i_{k}}\right)\left(\hat{\mathbf{b}}_{i_{k}}-\mathbf{b}_{i_{k}}\right)^{H}\right]$. By using the channel estimation model in (2), the averaged MSE-matrix results:

$$
\begin{gathered}
\overline{\mathbf{E}}_{i_{k}}\left(\mathbf{R}_{i_{k}},\left\{\mathbf{T}_{i_{k}}\right\}\right)=\mathbf{I}+\mathbf{R}_{i_{k}}^{H}\left(\sum_{j=1}^{I_{k}} \hat{\mathbf{H}}_{k, i_{k}} \mathbf{T}_{j_{k}} \mathbf{T}_{j_{k}}^{H} \hat{\mathbf{H}}_{k, i_{k}}^{H}+\mathbf{N}_{i_{k}}\right) \mathbf{R}_{i_{k}} \\
-\mathbf{R}_{i_{k}}^{H} \hat{\mathbf{H}}_{k, i_{k}} \mathbf{T}_{i_{k}}-\mathbf{T}_{i_{k}}^{H} \hat{\mathbf{H}}_{k, i_{k}}^{H} \mathbf{R}_{i_{k}}+\bar{J}_{k, i_{k}} \operatorname{Tr}\left(\mathbf{T}_{i_{k}} \mathbf{T}_{i_{k}}^{H}\right) \mathbf{R}_{i_{k}}^{H} \mathbf{R}_{i_{k}},(13)
\end{gathered}
$$

being $\bar{J}_{k, i_{k}}$ shown in (4).

\section{Signal model for uplink (phase 2)}

Assuming perfect UL-DL propagation channel reciprocity (i.e. $\overleftarrow{\mathbf{H}}_{k, j_{l}}=\mathbf{H}_{k, j_{l}}^{T}, \forall k, \forall j, \forall l$ ), the received signal at the $k$-th SeNB in the UL transmission is given by:

$$
\overleftarrow{\mathbf{y}}_{k}=\sum_{i=1}^{I_{k}} \mathbf{H}_{k, i_{k}}^{T} \overleftarrow{\mathbf{T}}_{i_{k}} \overleftarrow{\mathbf{s}}_{i_{k}}+\underbrace{\sum_{l=1, l \neq k j=1}^{K} \sum_{k, j_{l}}^{I_{l}} \mathbf{H}_{j_{l}}^{T} \overleftarrow{\mathbf{s}}_{j_{l}}}_{\text {interference }}+\overleftarrow{\mathbf{v}}_{k}
$$

where $\overleftarrow{\mathbf{T}}_{i_{k}}$ denotes the transmit filter used at the $i_{k}$-th UE, $\overleftarrow{\mathbf{s}}_{i_{k}}$ the stream of independent symbols sent by the $i_{k}$-th UE, and $\overleftarrow{\mathbf{v}}_{k}$ denotes the UL noise with distribution $\overleftarrow{\mathbf{v}}_{k} \sim \mathcal{C N}\left(\mathbf{0}, \overleftarrow{\sigma}_{k}^{2} \mathbf{I}\right)$. Then, the covariance matrix of the received signal at the $k$-th $\operatorname{SeNB} \mathbf{C}_{\overleftarrow{\mathbf{y}}_{k}}$ is given by:

$$
\mathbf{C}_{\overleftarrow{\mathbf{y}}_{k}}=\mathbb{E} \overleftarrow{\mathbf{s}}, \overleftarrow{\mathbf{v}}\left[\overleftarrow{\mathbf{y}}_{k} \overleftarrow{\mathbf{y}}_{k}^{H}\right]=\sum_{i=1}^{I_{k}} \mathbf{H}_{k, i_{k}}^{T} \overleftarrow{\mathbf{T}}_{i_{k}} \overleftarrow{\mathbf{T}}_{i_{k}}^{H} \mathbf{H}_{k, i_{k}}^{*}+\overleftarrow{\mathbf{N}}_{k}
$$

where $\overleftarrow{\mathbf{N}}_{k}$ denotes the covariance matrix of the received interference plus noise at the $k$-th SeNB:

$$
\overleftarrow{\mathbf{N}}_{k}=\sum_{l=1, l \neq k}^{K} \sum_{j=1}^{I_{l}} \mathbf{H}_{k, j_{l}}^{T} \overleftarrow{\mathbf{T}}_{j_{l}} \overleftarrow{\mathbf{T}}_{j_{l}}^{H} \mathbf{H}_{k, j_{l}}^{*}+\overleftarrow{\sigma}_{k}^{2} \mathbf{I}
$$

\section{Weighted Sum-Rate Maximization}

With the objective of maximizing the total DL weighted sum-rate (WSR) of the system with a maximum transmitted power constraint per SeNB, the transmit filters at SeNBs (or, equivalently, the transmit precoding matrices) are designed as the solution to the following optimization problem:

$$
\begin{aligned}
\left(\mathrm{P}_{\mathrm{WSR}}\right): & \underset{\left\{\mathbf{T}_{i_{k}}\right\}_{\forall k, \forall i}}{\operatorname{maximize}} \sum_{k=1}^{K} \sum_{i=1}^{I_{k}} \mu_{i_{k}} R_{i_{k}}\left(\left\{\mathbf{T}_{i_{k}}\right\}\right) \\
& \text { subject to } \quad \sum_{i=1}^{I_{k}} \operatorname{Tr}\left(\mathbf{T}_{i_{k}} \mathbf{T}_{i_{k}}^{H}\right) \leq P_{k}^{\mathrm{SeNB}} \quad \forall k,
\end{aligned}
$$

where $\mu_{i_{k}}$ is a weighting coefficient associated to the priority of the $i_{k}$-th UE, $R_{i_{k}}\left(\left\{\mathbf{T}_{i_{k}}\right\}\right)$ is the achievable rate in (12), and $P_{k}^{\mathrm{SeNB}}$ is the available transmit power at the $k$-th SeNB.

Due to interference, problem $\left(\mathrm{P}_{\mathrm{WSR}}\right)$ in (17) is not convex on $\left\{\mathbf{T}_{i_{k}}\right\}$ and the optimal solution cannot be guaranteed. Nevertheless, it is shown in [11] that one solution for transmit filters that attains a local optima of the maximum WSR problem $\left(\mathrm{P}_{\mathrm{WSR}}\right)$ in (17) can be obtained by solving the following optimization problem that considers minimization of the total sum of weighted mean square errors (WMSE):

$$
\begin{gathered}
\left(\mathrm{P}_{\text {WMSE }}\right): \underset{\begin{array}{c}
\left\{\mathbf{T}_{i_{k}}\right\},\left\{\mathbf{R}_{i_{k}}\right\}, \\
\left\{\mathbf{W}_{i_{k}}\right\} \forall k, \forall i
\end{array}}{\operatorname{minimize}}, \sum_{k=1}^{K} \sum_{i=1}^{I_{k}}\left(\operatorname{Tr}\left(\mathbf{W}_{i_{k}} \mathbf{E}_{i_{k}}\left(\mathbf{R}_{i_{k}},\left\{\mathbf{T}_{i_{k}}\right\}\right)\right)\right. \\
\left.\quad-\mu_{i_{k}} \log _{2}\left|\mu_{i_{k}}^{-1} \mathbf{W}_{i_{k}}\right|\right) \\
\text { subject to } \quad \sum_{i=1}^{I_{k}} \operatorname{Tr}\left(\mathbf{T}_{i_{k}} \mathbf{T}_{i_{k}}^{H}\right) \leq P_{k}^{\mathrm{SeNB}} \quad \forall k,
\end{gathered}
$$

where $\mathbf{W}_{i_{k}}$ is a weighting matrix associated to the $i_{k}$-th UE and $\mathbf{E}_{i_{k}}\left(\mathbf{R}_{i_{k}},\left\{\mathbf{T}_{i_{k}}\right\}\right)$ corresponds to the MSE-matrix in (11). 
Problem $\left(\mathrm{P}_{\mathrm{WMSE}}\right)$ in $(18)$ is not jointly convex on all sets of optimization variables, but it turns out to be convex for each set of unknowns $\left(\left\{\mathbf{T}_{i_{k}}\right\},\left\{\mathbf{R}_{i_{k}}\right\}\right.$, and $\left.\left\{\mathbf{W}_{i_{k}}\right\}\right)$ separately. Furthermore, each of them can be derived analytically assuming that the other two sets of variables are fixed. Therefore, a block coordinate descent approach [16] can be followed to find a local optimum of the problem $\left(\mathrm{P}_{\mathrm{WMSE}}\right)$ in (18) by alternate optimization of transmit filters $\left\{\mathbf{T}_{i_{k}}\right\}$, receive filters $\left\{\mathbf{R}_{i_{k}}\right\}$, and weighting matrices $\left\{\mathbf{W}_{i_{k}}\right\}$. The attained solution is a local optimal solution of the maximum WSR problem $\left(\mathrm{P}_{\mathrm{WSR}}\right)$ in (17) (see details in [11]).

In case a centralized procedure is implemented, it is required that all channel matrices from all SeNBs to all UEs (i.e. $\left.\left\{\mathbf{H}_{k, j_{l}}\right\}, \forall k, \forall j, \forall l\right)$ are collected in a central processor node. The decentralized implementation proposed in [11], where $\left\{\mathbf{T}_{i_{k}}\right\}$ are updated at SeNBs while $\left\{\mathbf{R}_{i_{k}}\right\}$ and $\left\{\mathbf{W}_{i_{k}}\right\}$ are updated at UEs, also requires that each $k$-th SeNB knows the channel matrices towards all UEs in the network (i.e. $\left\{\mathbf{H}_{k, j_{l}}\right\}$, $\forall j, \forall l)$ and that feedback links are available from each UEs towards all SeNBs to report the updated $\mathbf{R}_{i_{k}}$ and $\mathbf{W}_{i_{k}}$.

\section{Decentralized Coordinated Precoding}

Different from [9]-[11], in this section a decentralized coordinated precoding (D-CoP) design for maximizing the WSR in (17) with linear transmit/receive filters is presented. First, through the use of the minimum WMSE problem $\left(\mathrm{P}_{\mathrm{WMSE}}\right)$ in (18) the maximum WSR problem is decomposed into parallel subproblems (one per SeNB) and a decentralized solution for DL transmit filters design at SeNBs is derived, either assuming perfect CSI of the direct channel matrices in subsection IV-A1 or assuming imperfect CSI conditions in subsection IV-A2. Subsection IV-B details the receive filter to be used at each UE. Second, how to acquire the required parameters for transmit filters design at each SeNB is detailed: in subsection IV-C the acquisition of the inter-cell interference plus noise covariance matrices $\left(\left\{\mathbf{N}_{i_{k}}\right\}, \forall i\right)$ is shown, and subsection IV-D describes how to acquire the interference-cost matrix $\left(\boldsymbol{\Upsilon}_{k}\right)$ by exploiting an UL transmission. Finally, the iterative algorithm for D$\mathrm{CoP}$ is presented in Section $\mathrm{V}$, subsuming the acquisition of the required parameters at each SeNB from the UL and the simultaneous per-SeNB optimizations for DL, as is shown in Fig. 2.

Let us define the following matrix that will allow us to decompose problem $\left(\mathrm{P}_{\mathrm{WMSE}}\right)$ in (18):

$$
\boldsymbol{\Upsilon}_{k}=\sum_{l=1, l \neq k}^{K} \sum_{j=1}^{I_{l}} \mathbf{H}_{k, j_{l}}^{H} \mathbf{R}_{j_{l}} \mathbf{W}_{j_{l}} \mathbf{R}_{j_{l}}^{H} \mathbf{H}_{k, j_{l}},
$$

which is referred to as the interference-cost matrix because it reflects the interference that can be created by the $k$-th SeNB towards unintended UEs (i.e. $j_{l}, l \neq k$ ) and it is seen as a penalizing term for the design of transmit filters at the $k$-th SeNB (see next problem in (20)).

Proposition 1: Problem ( $\mathrm{P}_{\mathrm{WMSE}}$ ) in (18) can be decomposed into $K$ parallel optimization problems (one per SeNB), where the problem to be solved at the $k$-th SeNB for a fixed

$$
\begin{aligned}
& \Upsilon_{k} \text { and }\left\{\mathbf{N}_{i_{k}}\right\}, \forall i \text {, is: } \\
& \left(\mathrm{P}_{\text {WMSE }}^{k}\right): \underset{\substack{\left\{\mathbf{T}_{i_{k}}\right\},\left\{\mathbf{R}_{i_{k}}\right\} \\
\left\{\mathbf{W}_{i_{k}}\right\}_{\forall i}}}{\operatorname{minimize}} \sum_{i=1}^{I_{k}}\left(\operatorname{Tr}\left(\mathbf{W}_{i_{k}} \mathbf{E}_{i_{k}}\left(\mathbf{R}_{i_{k}},\left\{\mathbf{T}_{i_{k}}\right\}, \mathbf{N}_{i_{k}}\right)\right)\right. \\
& \left.-\mu_{i_{k}} \log _{2}\left|\mu_{i_{k}}^{-1} \mathbf{W}_{i_{k}}\right|+\operatorname{Tr}\left(\boldsymbol{\Upsilon}_{k} \mathbf{T}_{i_{k}} \mathbf{T}_{i_{k}}^{H}\right)\right) \\
& \text { subject to } \sum_{i=1}^{I_{k}} \operatorname{Tr}\left(\mathbf{T}_{i_{k}} \mathbf{T}_{i_{k}}^{H}\right) \leq P_{k}^{\mathrm{SeNB}},
\end{aligned}
$$

where $\mathbf{E}_{i_{k}}\left(\mathbf{R}_{i_{k}},\left\{\mathbf{T}_{i_{k}}\right\}, \mathbf{N}_{i_{k}}\right)$ is the MSE-matrix in (11) assuming that $\mathbf{N}_{i_{k}}$ is fixed.

Proof: See Appendix A, where it is shown that the gradients of problem $\left(\mathrm{P}_{\mathrm{WMSE}}\right)$ in (18) and problem $\left(\mathrm{P}_{\mathrm{WMSE}}^{k}\right)$ in (20) with respect to $\left\{\mathbf{T}_{i_{k}}\right\},\left\{\mathbf{R}_{i_{k}}\right\},\left\{\mathbf{W}_{i_{k}}\right\}, \forall i$, are the same if $\boldsymbol{\Upsilon}_{k}$ and $\left\{\mathbf{N}_{i_{k}}\right\}, \forall i$, are fixed.

\section{A. Transmit filters design at each SeNB}

1) Perfect CSI case: Problem ( $\left.\mathrm{P}_{\mathrm{WMSE}}^{k}\right)$ in (20) is convex on each set of variables separately, and each of them can be derived analytically assuming that the other two sets are fixed. Therefore, each $k$-th SeNB can solve problem ( $\mathrm{P}_{\mathrm{WMSE}}^{k}$ ) in (20) for given $\boldsymbol{\Upsilon}_{k}$ and $\left\{\mathbf{N}_{i_{k}}\right\}, \forall i$, with alternate optimization among the following three sets of variables:

$$
\begin{aligned}
\text { i }: \mathbf{R}_{i_{k}}^{(n)} & =\left(\mathbf{A}_{i_{k}}^{(n-1)}+\mathbf{N}_{i_{k}}\right)^{-1} \mathbf{H}_{k, i_{k}} \mathbf{T}_{i_{k}}^{(n-1)}, \forall i, \\
\text { ii : } \mathbf{W}_{i_{k}}^{(n)} & =\mu_{i_{k}} \mathbf{E}_{i_{k}}^{-1}\left(\mathbf{R}_{i_{k}}^{(n)},\left\{\mathbf{T}_{i_{k}}^{(n-1)}\right\}, \mathbf{N}_{i_{k}}\right), \forall i, \\
\text { iii : } \mathbf{T}_{i_{k}}^{(n)} & =\left(\mathbf{B}_{k}^{(n)}+\mathbf{\Upsilon}_{k}+\lambda_{k}^{(n)} \mathbf{I}\right)^{-1} \mathbf{H}_{k, i_{k}}^{H} \mathbf{R}_{i_{k}}^{(n)} \mathbf{W}_{i_{k}}^{(n)}, \forall i,
\end{aligned}
$$

where

$$
\begin{gathered}
\mathbf{A}_{i_{k}}^{(n-1)}=\sum_{j=1}^{I_{k}} \mathbf{H}_{k, i_{k}} \mathbf{T}_{j_{k}}^{(n-1)} \mathbf{T}_{j_{k}}^{(n-1) H} \mathbf{H}_{k, i_{k}}^{H}, \\
\mathbf{B}_{k}^{(n)}=\sum_{j=1}^{I_{k}} \mathbf{H}_{k, j_{k}}^{H} \mathbf{R}_{j_{k}}^{(n)} \mathbf{W}_{j_{k}}^{(n)} \mathbf{R}_{j_{k}}^{(n) H} \mathbf{H}_{k, j_{k}},
\end{gathered}
$$

$n$ is the iteration number, and $\lambda_{k}^{(n)}$ denotes a non-negative dual variable associated to the per-SeNB power constraint in (20). At each iteration, $\lambda_{k}^{(n)}$ has to be optimized to meet the power constraint, which can be efficiently solved using convex optimization techniques (e.g. subgradient method) [17].

Hence, monotonic convergence of the decentralized problem $\left(\mathrm{P}_{\mathrm{WMSE}}^{k}\right)$ in (20) is ensured for given $\boldsymbol{\Upsilon}_{k}$ and $\left\{\mathbf{N}_{i_{k}}\right\}, \forall i$. However, due to inter-cell interference, so far we cannot guarantee that if each $k$-th SeNB solves its own problem $\left(\mathrm{P}_{\mathrm{WMSE}}^{k}\right)$ in (20) and all of them do it simultaneously, convergence of $\left(\mathrm{P}_{\mathrm{WMSE}}\right)$ in (18) is achieved.

Let us recall that the proposed solution for DL transmit filters design in (21) differs from [11], where each SeNB updates the transmit filters $\left\{\mathbf{T}_{i_{k}}\right\}, \forall i$, by following the last equation in (21) and evaluating the expression of $\boldsymbol{\Upsilon}_{k}$ in (19), which requires knowledge of all channel matrices, all weighting matrices, and all receive filters used at all UEs (i.e. $\mathbf{H}_{k, j_{l}}, \mathbf{W}_{j_{l}}$ and $\left.\mathbf{R}_{j_{l}}, \forall j, \forall l\right)$. 
2) Imperfect CSI case: When the direct channel matrices towards the served UEs (i.e. $\left\{\mathbf{H}_{k, i_{k}}\right\}, \forall i$ ) are acquired with errors at the $k$-th SeNB, a stochastic (or Bayesian) robust design [15] can be used to overcome the impact of channel estimation errors. In our case, so as to implement a robust DL transmit filters design, the averaged MSE-matrix $\overline{\mathbf{E}}_{i_{k}}\left(\mathbf{R}_{i_{k}},\left\{\mathbf{T}_{i_{k}}\right\}, \mathbf{N}_{i_{k}}\right)$ in (13) (assuming that $\mathbf{N}_{i_{k}}$ is fixed) has to be considered under the assumption that $\hat{\mathbf{H}}_{k, i_{k}}$ in (3) and $\bar{J}_{k, i_{k}}$ in (4), $\forall i$, are known at the $k$-th SeNB. Therefore, the decentralized problem to be solved at the $k$-th SeNB is $\left(\mathrm{P}_{\mathrm{WMSE}}^{k}\right)$ in (20) with $\overline{\mathbf{E}}_{i_{k}}\left(\mathbf{R}_{i_{k}},\left\{\mathbf{T}_{i_{k}}\right\}, \mathbf{N}_{i_{k}}\right)$ instead of $\mathbf{E}_{i_{k}}\left(\mathbf{R}_{i_{k}},\left\{\mathbf{T}_{i_{k}}\right\}, \mathbf{N}_{i_{k}}\right)$. Similarly as for the perfect CSI case in (20), the problem is convex on each set of variables separately, and each of them can be derived analytically assuming that the other two sets are fixed. Therefore, each $k$-th SeNB can find a robust transmit filters design for given $\boldsymbol{\Upsilon}_{k}$ and $\left\{\mathbf{N}_{i_{k}}\right\}, \forall i$, with alternate optimization among the following three sets of variables:

$$
\begin{aligned}
\text { i }: \mathbf{R}_{i_{k}}^{(n)} & =\left(\mathbf{A}_{i_{k}}^{(n-1)}+\mathbf{N}_{i_{k}}\right)^{-1} \hat{\mathbf{H}}_{k, i_{k}} \mathbf{T}_{i_{k}}^{(n-1)}, \forall i, \\
\text { ii : } \mathbf{W}_{i_{k}}^{(n)} & =\mu_{i_{k}} \overline{\mathbf{E}}_{i_{k}}^{-1}\left(\mathbf{R}_{i_{k}}^{(n)},\left\{\mathbf{T}_{i_{k}}^{(n-1)}\right\}, \mathbf{N}_{i_{k}}\right), \forall i, \\
\text { iii : } \mathbf{T}_{i_{k}}^{(n)} & =\left(\mathbf{B}_{k}^{(n)}+\mathbf{\Upsilon}_{k}+\lambda_{k}^{(n)} \mathbf{I}\right)^{-1} \hat{\mathbf{H}}_{k, i_{k}}^{H} \mathbf{R}_{i_{k}}^{(n)} \mathbf{W}_{i_{k}}^{(n)}, \forall i,
\end{aligned}
$$

where

$$
\begin{aligned}
& \mathbf{A}_{i_{k}}^{(n-1)}=\sum_{j=1}^{I_{k}}\left(\hat{\mathbf{H}}_{k, i_{k}} \mathbf{T}_{j_{k}}^{(n-1)} \mathbf{T}_{j_{k}}^{(n-1) H} \hat{\mathbf{H}}_{k, i_{k}}^{H}\right.\left.+\bar{J}_{k, j_{k}} \operatorname{Tr}\left(\mathbf{T}_{j_{k}}^{(n-1)} \mathbf{T}_{j_{k}}^{(n-1) H}\right) \mathbf{I}\right), \\
& \mathbf{B}_{k}^{(n)}=\sum_{j=1}^{I_{k}}\left(\hat{\mathbf{H}}_{k, j_{k}}^{H} \mathbf{R}_{j_{k}}^{(n)} \mathbf{W}_{j_{k}}^{(n)} \mathbf{R}_{j_{k}}^{(n) H} \hat{\mathbf{H}}_{k, j_{k}}\right. \\
&\left.+\bar{J}_{k, j_{k}} \operatorname{Tr}\left(\mathbf{R}_{j_{k}}^{(n)} \mathbf{W}_{j_{k}}^{(n)} \mathbf{R}_{j_{k}}^{(n) H}\right) \mathbf{I}\right),
\end{aligned}
$$

$n$ is the iteration number, $\overline{\mathbf{E}}_{i_{k}}\left(\mathbf{R}_{i_{k}},\left\{\mathbf{T}_{i_{k}}\right\}, \mathbf{N}_{i_{k}}\right)$ is the averaged MSE-matrix in (13) assuming $\mathbf{N}_{i_{k}}$ is fixed, and $\lambda_{k}^{(n)}$ denotes the non-negative dual variable associated to the per-SeNB power constraint in (20) (to be optimized at each iteration).

\section{B. Receive filter design at each $U E$}

Given the transmit filters, $\left\{\mathbf{T}_{i_{k}}\right\}$, the optimal DL receive filters for each $i_{k}$-th UE are given by the minimum MSE (MMSE) receivers [12]:

$$
\mathbf{R}_{i_{k}}=\mathbf{C}_{\mathbf{y}_{i_{k}}}^{-1} \mathbf{H}_{k, i_{k}} \mathbf{T}_{i_{k}},
$$

where $\mathbf{C}_{\mathbf{y}_{i_{k}}}$ is defined in (8). As it is done in real deployments [18], each UE can compute $\mathbf{R}_{i_{k}}$ in (23) based on the estimation of the equivalent channel $\mathbf{H}_{k, i_{k}} \mathbf{T}_{i_{k}}$ and the covariance matrix of the received signal $\mathbf{C}_{\mathbf{y}_{i_{k}}}$. Then, each UE can also compute the weighting matrix $\mathbf{W}_{i_{k}}=\mu_{i_{k}} \mathbf{E}_{i_{k}}^{-1}$ based on $\mathbf{C}_{\mathbf{y}_{i_{k}}}, \mathbf{H}_{k, i_{k}} \mathbf{T}_{i_{k}}$, and $\mathbf{R}_{i_{k}}$ using (11).

\section{Acquisition of $\boldsymbol{N}_{i_{k}}, \forall i$, at each $\mathrm{SeNB}$}

We assume that $\mathbf{C}_{\mathbf{y}_{i_{k}}}$ is reported from the UE towards the serving SeNB through an UL feedback link (in Phase 2 UL in Fig 2), such that the SeNB collects $\mathbf{C}_{\mathbf{y}_{i_{k}}}, \forall i$, from its served UEs. Let us recall that, as compared to [11] where feedback links are required from each UE towards all SeNBs in the network in order to report $\mathbf{R}_{i_{k}}$ and $\mathbf{W}_{i_{k}}$, in our case only one feedback link is needed from each UE towards the serving SeNB through which only $\mathbf{C}_{\mathbf{y}_{i_{k}}}$ is reported. To reduce the communication overhead, the fact that $\mathbf{C}_{\mathbf{y}_{i_{k}}}$ is a positive semidefinite matrix (and hence hermitian) can be exploited.

Therefore, the inter-cell interference plus noise covariance matrices $\left\{\mathbf{N}_{i_{k}}\right\}, \forall i$, can be easily estimated at the $k$-th SeNB based on $\left\{\mathbf{H}_{k, i_{k}}\right\},\left\{\mathbf{T}_{i_{k}}\right\}$, and $\left\{\mathbf{C}_{\mathbf{y}_{i_{k}}}\right\}, \forall i$, by following (8):

$$
\mathbf{N}_{i_{k}}=\mathbf{C}_{\mathbf{y}_{i_{k}}}-\sum_{j=1}^{I_{k}} \mathbf{H}_{k, i_{k}} \mathbf{T}_{j_{k}} \mathbf{T}_{j_{k}}^{H} \mathbf{H}_{k, i_{k}}^{H} .
$$

So it is not needed to estimate the interfering channel matrices (i.e. $\mathbf{H}_{l, i_{k}}, \forall l \neq k$ ) to compute $\mathbf{N}_{i_{k}}$ in (9) at SeNBs.

\section{Acquisition of $\boldsymbol{\Upsilon}_{k}$ at each SeNB}

In order to avoid the complex task of estimating the most harmful interfering channel matrices that are needed to compute the interference-cost matrix $\Upsilon_{k}$ in (19) (as is assumed in [11]), we propose obtaining an estimate of $\Upsilon_{k}$ from the covariance matrix of the UL interference plus noise (i.e. $\overleftarrow{\mathbf{N}}_{k}$ in (16)) when properly designing UL transmit filters $\overleftarrow{\mathbf{T}}_{j_{l}}$ (see Phase 2 - UL in Fig 2). By doing so, estimation of interfering channel matrices is avoided as $\overline{\mathbf{N}}_{k}$ can be computed by subtracting the desired signals to the covariance matrix of the UL received signal at the $k$-th SeNB (see (15)).

Notice that $\overleftarrow{\mathbf{N}}_{k}^{*}$ in (16) and $\boldsymbol{\Upsilon}_{k}$ in (19) differ just in the noise term in case UEs employ as UL transmit filter $\overleftarrow{\mathbf{T}}_{j_{l}}=\mathbf{R}_{j_{l}}^{*} \mathbf{W}_{j_{l}}^{\frac{1}{2} *}, \forall j, \forall l$, where $\mathbf{W}_{j_{l}}=\mathbf{W}_{j_{l}}^{\frac{1}{2}} \mathbf{W}_{j_{l}}^{\frac{1}{2} H}$. Therefore in case the available power at UEs is not a limiting factor, by using a precoded UL pilot-based transmission, we could have a biased estimate of $\boldsymbol{\Upsilon}_{k}$ as: $\hat{\Upsilon}_{k}=\overleftarrow{\mathbf{N}}_{k}^{*}=\boldsymbol{\Upsilon}_{k}+\overleftarrow{\sigma}_{k}^{2} \mathbf{I}$.

Nevertheless, usually UL transmit filters cannot be applied as such unless we take into account the maximum transmit power constraints for UL, i.e.

$$
\operatorname{Tr}\left(\overleftarrow{\mathbf{T}}_{j_{l}} \overleftarrow{\mathbf{T}}_{j_{l}}^{H}\right) \leq P_{j_{l}}^{\mathrm{UE}}
$$

where $P_{j_{l}}^{\mathrm{UE}}$ is the available power at the $j_{l}$-th UE. In this regard, the proposed solution is to scale the DL receive filters in (23) by a common scaling cell-wide factor $F<1$ as: $\overleftarrow{\mathbf{T}}_{j_{l}}=\sqrt{F} \mathbf{R}_{j_{l}}^{*} \mathbf{W}_{j_{l}}^{\frac{1}{2} *}$. The scaling cell-wide factor $F$ would be designed beforehand in order to ensure that the $\epsilon \%$ of the UEs fulfill the transmit power constraint in (25), i.e. $\operatorname{Pr}\left(\operatorname{Tr}\left(F \mathbf{R}_{j_{l}}^{*} \mathbf{W}_{j_{l}}^{*} \mathbf{R}_{j_{l}}^{T}\right) \leq P_{j_{l}}^{\mathrm{UE}}\right)=\epsilon$. However, it is assumed that those UEs not satisfying the constraint in (25) with the a priori selected $F$ will transmit at maximum power by scaling the DL receive filters with a per-user factor $f_{j_{l}}<F$. So, the proposed UL transmit filter is:

$$
\overleftarrow{\mathbf{T}}_{j_{l}}=\sqrt{f_{j_{l}}} \mathbf{R}_{j_{l}}^{*} \mathbf{W}_{j_{l}}^{\frac{1}{2} *},
$$

where

$$
f_{j_{l}}= \begin{cases}F, & \text { if } \operatorname{Tr}\left(F \mathbf{R}_{j_{l}}^{*} \mathbf{W}_{j_{l}}^{*} \mathbf{R}_{j_{l}}^{T}\right) \leq P_{j_{l}}^{\mathrm{UE}}, \\ \frac{P_{j_{l}}^{\mathrm{UE}}}{\operatorname{Tr}\left(\mathbf{R}_{j_{l}}^{*} \mathbf{W}_{j_{l}}^{*} \mathbf{R}_{j_{l}}^{T}\right)}, & \text { otherwise. }\end{cases}
$$


By using this approach, we can obtain a biased estimate of $\Upsilon_{k}$ in (19) from the covariance matrix of the UL interference plus noise $\overleftarrow{\mathbf{N}}_{k}$ in (16). Its expected value is:

$$
\begin{aligned}
\hat{\boldsymbol{\Upsilon}}_{k}=\frac{1}{F} \overleftarrow{\mathbf{N}}_{k}^{*} & =\sum_{l=1, l \neq k}^{K} \sum_{j=1}^{I_{l}} \frac{f_{j_{l}}}{F} \mathbf{H}_{k, j_{l}}^{H} \mathbf{R}_{j_{l}} \mathbf{W}_{j_{l}} \mathbf{R}_{j_{l}}^{H} \mathbf{H}_{k, j_{l}}+\frac{\overleftarrow{\sigma}_{k}^{2}}{F} \mathbf{I} \\
& =\mathbf{\Upsilon}_{k}+\tilde{\boldsymbol{\Upsilon}}_{k}+\frac{1}{F} \overleftarrow{\sigma}_{k}^{2} \mathbf{I},
\end{aligned}
$$

where $\tilde{\Upsilon}_{k}$ describes the error introduced by those UEs served by neighbor SeNBs $(l \neq k)$ that are transmitting at maximum power (i.e. $f_{j_{l}}<F$ ):

$$
\tilde{\boldsymbol{\Upsilon}}_{k}=\sum_{l=1, l \neq k}^{K} \sum_{j=1}^{I_{l}} \frac{1}{F}\left(f_{j_{l}}-F\right) \mathbf{H}_{k, j_{l}}^{H} \mathbf{R}_{j_{l}} \mathbf{W}_{j_{l}} \mathbf{R}_{j_{l}}^{H} \mathbf{H}_{k, j_{l}} .
$$

The proposed approach presents a bias that depends on the noise power increased by $F^{-1}$ and an additional matrix associated to the non-serving UEs that are transmitting at maximum power (see (28)). So, there is an evident trade-off when the UE transmit power is a limiting factor: if $F$ is small, the errors in the estimation of $\boldsymbol{\Upsilon}_{k}$ in (28) come due to the increased noise power, while if $F$ is large the errors come due to $\tilde{\Upsilon}_{k}$ in (29) as the major part of the UEs will use a peruser scaling factor $f_{j_{l}}$ lower than $F$ (see (26)). However, as is shown in [1], the estimation errors in $\hat{\Upsilon}_{k}$ are negligible and do not affect the system performance when properly selecting the scaling cell-wide factor $F$ in (26) in such a way that a certain percentage of the UEs (which depends on the deployment) fulfill the transmit power constraint in (25) with $f_{j_{l}}=F$. The intuitive explanation for a SISO case comes in the following: the UEs highly interfered in the DL have a lower absolute value of the receive filter in the DL due to the interference (see (21)) such that they would use $f_{j_{l}}=F$ as scaling factor in (26). This way, no errors would appear from the contributions of the highly interfered users to (28) and, as they are the users which contribute more to (28), the errors from the other users would be negligible. The key point is the selection of the scaling cell-wide factor $F$ to allow a group of UEs to fulfill the constraint, while not making it too small such that the contribution of the noise power predominates in (28).

Note that all what is needed to obtain $\hat{\Upsilon}_{k}$ in (28) from the UL transmission is the received signal covariance matrix, which can be obtained from the coordinated use of UL pilots.

We would like to remark that the proposed scheme for estimating the interference-cost matrix $\boldsymbol{\Upsilon}_{k}$ avoids the estimation errors of the interfering channel matrices that comes up in the procedure proposed in [11]. In such a case, since every single channel matrix has to be estimated, the robust transmit filters design in subsection IV-A2 should use the following matrix $\boldsymbol{\Phi}_{k}$ instead of $\boldsymbol{\Upsilon}_{k}$ in (22):

$$
\mathbf{\Phi}_{k}=\sum_{\substack{l=1 \\ l \neq k}}^{K} \sum_{j=1}^{I_{l}}\left(\hat{\mathbf{H}}_{k, j_{l}}^{H} \mathbf{R}_{j_{l}} \mathbf{W}_{j_{l}} \mathbf{R}_{j_{l}}^{H} \hat{\mathbf{H}}_{k, j_{l}}+\bar{J}_{k, j_{l}} \operatorname{Tr}\left(\mathbf{R}_{j_{l}} \mathbf{W}_{j_{l}} \mathbf{R}_{j_{l}}^{H}\right) \mathbf{I}\right),
$$

which includes the estimation errors of the interfering channel matrices from the $k$-th SeNB towards all the unintended UEs (i.e. $j_{l}, l \neq k$ ). Nevertheless, in the proposed D-CoP an
TABLE I: INTERFERENCE-COST MATRIX ACQUiSITION

\begin{tabular}{llc}
\hline \hline & Perfect CSI & Imperfect CSI \\
D-WMMSE in [11] & $\boldsymbol{\Upsilon}_{k}$ in (19) & $\boldsymbol{\Phi}_{k}$ in (30) \\
Proposed D-CoP & $\hat{\boldsymbol{\Upsilon}}_{k}$ in (28) & $\hat{\boldsymbol{\Upsilon}}_{k}$ in (28) \\
\hline \hline
\end{tabular}

estimate of $\boldsymbol{\Upsilon}_{k}$ can be obtained from the UL transmission as a function of the real channel matrices (see (28)), hence avoiding channel estimation errors of the interfering links and resulting in enhanced robustness. In this regard, Table I summarizes the interference-cost matrix that has to be used for transmit coordination depending on whether the interfering channels are estimated (as in the decentralized procedure in [11]) or not (as in the proposed D-CoP). Further, in case a centralized approach was used then $\mathbf{N}_{i_{k}}$ in (22) should also include the estimation errors from all SeNBs $(l \neq k)$ towards the $i_{k}$-th $\mathrm{UE}$, resulting even in a worse performance.

\section{Algorithm FOR D-COP}

Algorithm in Table II summarizes the iterative procedure to solve $\left(\mathrm{P}_{\mathrm{WMSE}}\right)$ in (18) in a decentralized manner. The procedure follows the phases shown in Fig. 2. During the channel estimation phase (Phase 1), the direct channel matrices $\mathbf{H}_{k, i_{k}}, \forall i$, are acquired at each $k$-th SeNB, which remain constant during the optimization (Phase 2) where the iterative algorithm for D-CoP is implemented and DL/UL transmissions are duplexed. The algorithm for D-CoP (Phase 2) includes the acquisition of the required parameters in UL (i.e. $\mathbf{N}_{i_{k}}$ as is detailed in subsection IV-C, $\forall i$, and $\Upsilon_{k}$ as is shown in subsection IV-D) and the simultaneous transmit filters designs that are performed at each SeNB for DL (i.e. (21) in subsection IV-A1 for perfect CSI or (22) in subsection IV-A2 for imperfect CSI).

$N_{\text {iter }}$ denotes the number of iterations allowed, and $t$ the iteration index. The algorithm starts from an initialization of the transmit filters $\left\{\mathbf{T}_{i_{k}}^{(0)}\right\}, \forall i$, at each $k$-th SeNB that satisfy the per-SeNB power constraint in (20). For simulation purposes, a suitable initialization of $\left\{\mathbf{T}_{i_{k}}^{(0)}\right\}, \forall i$, can be obtained by solving $\left(\mathrm{P}_{\mathrm{WMSE}}^{k}\right)$ in (20) using $\mathbf{\Upsilon}_{k}^{(0)}=\mathbf{0}$. Then, a DL transmission is carried out using $\left\{\mathbf{T}_{i_{k}}^{(0)}\right\}$ (Phase 2 - DL(0)), where UEs can evaluate the covariance matrix of the received signal $\mathbf{C}_{\mathbf{y}_{i_{k}}}$ and update the receive filter $\mathbf{R}_{i_{k}}^{(0)}$ and the weighting matrix $\mathbf{W}_{i_{k}}^{(0)}$ using (23) (line 3). See details in subsection IV-B.

Then, at each iteration $t=1, \ldots, N_{\text {iter }}$, the following steps are followed:

- Feedback of $\mathbf{C}_{\mathbf{y}_{i_{k}}}^{(t-1)}$ to acquire $\mathbf{N}_{i_{k}}^{(t-1)}$ at SeNB (Phase 2 - UL $(t)$ ): Each UE reports $\mathbf{C}_{\mathbf{y}_{i_{k}}}^{(t-1)}$ to the serving SeNB (line 5), and hence the SeNB can acquire the intercell interference plus noise covariance matrix $\mathbf{N}_{i_{k}}^{(t-1)}$ of the served UEs using (24), $\forall i$ (line 6). See details in subsection IV-C.

- UL transmission to acquire $\hat{\Upsilon}_{k}^{(t)}$ at SeNB (Phase 2 $\mathrm{UL}(t))$ : An UL transmission is carried out, where all UEs transmit with $\overleftarrow{\mathbf{T}}_{i_{k}}^{(t)}$ in (26) computed from $\mathbf{R}_{i_{k}}^{(t-1)}$ and $\mathbf{W}_{i_{k}}^{(t-1)}$ (line 7), such that each SeNB can acquire 
TABle II: D-CoP Procedure to Solve ( $\mathrm{P}_{\text {Wmse }}$ ) in (18) with Simultaneous Per-SeNB Optimizations

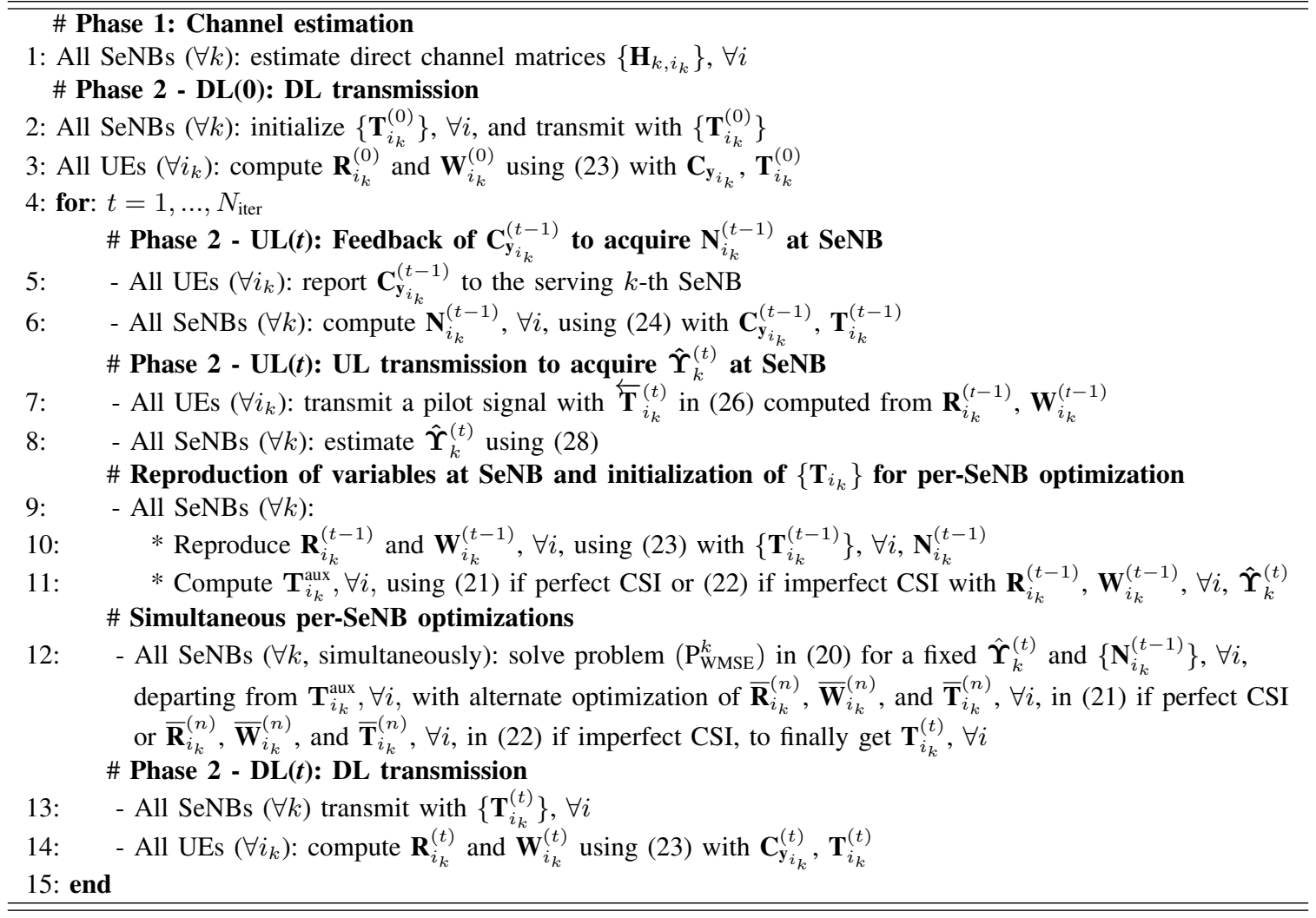

the interference-cost matrix $\hat{\Upsilon}_{k}^{(t)}$ using (28) (line 8). See details in subsection IV-D.

- Reproduction of variables at SeNB and initialization of $\left\{\mathbf{T}_{i_{k}}\right\}$ for per-SeNB optimization: Each SeNB reproduces $\left\{\mathbf{R}_{i_{k}}^{(t-1)}\right\}$ and $\left\{\mathbf{W}_{i_{k}}^{(t-1)}\right\}, \forall i$, based on the acquired $\left\{\mathbf{N}_{i_{k}}^{(t-1)}\right\}, \forall i$, and the transmit filters used in the previous iteration $\left\{\mathbf{T}_{i_{k}}^{(t-1)}\right\}, \forall i$ (line 10). Note that $\left\{\mathbf{R}_{i_{k}}^{(t-1)}\right\}$ and $\left\{\mathbf{W}_{i_{k}}^{(t-1)}\right\}$ coincide with the ones computed at the served UEs in the previous iteration if $\mathbf{C}_{\mathbf{y}_{i_{k}}}^{(t-1)}$ is perfectly reported. Then, with the reproduced $\left\{\mathbf{R}_{i_{k}}^{(t-1)}\right\}$ and $\left\{\mathbf{W}_{i_{k}}^{(t-1)}\right\}, \forall i$, each SeNB can update the transmit filters $\left\{\mathbf{T}_{i_{k}}\right\}, \forall i$, (denoted by $\left\{\mathbf{T}_{i_{k}}^{\text {aux }}\right\}, \forall i$ ) by using last equation in (21) if perfect CSI or in (22) if imperfect CSI (line 11). $\left\{\mathbf{T}_{i_{k}}^{\text {aux }}\right\}$ will be used as initialization for the optimization at SeNB.

- Simultaneous per-SeNB optimizations: By starting with $\left\{\mathbf{T}_{i_{k}}^{\text {aux }}\right\}, \forall i$, an alternate optimization is performed at each SeNB for fixed $\left\{\mathbf{N}_{i_{k}}^{(t-1)}\right\}, \forall i$, and $\hat{\boldsymbol{\Upsilon}}_{k}^{(t)}$ that have been acquired in the previous steps. Receive filters $\left\{\mathbf{R}_{i_{k}}\right\}$, weighting matrices $\left\{\mathbf{W}_{i_{k}}\right\}$, and transmit filters $\left\{\mathbf{T}_{i_{k}}\right\}, \forall i$, are iteratively computed by using (21) if perfect CSI or by using (22) if imperfect CSI (line 12). See subsections IV-A1 and IV-A2. The obtained transmit filters design are denoted by $\left\{\mathbf{T}_{i_{k}}^{(t)}\right\}$.

- DL transmission (Phase 2 - DL $(t)$ ): DL transmission is carried out using $\left\{\mathbf{T}_{i_{k}}^{(t)}\right\}$ (line 13), where UEs can evaluate the covariance matrix of the received signal $\mathbf{C}_{\mathbf{y}_{i_{k}}}^{(t)}$ and update the receive filter $\mathbf{R}_{i_{k}}^{(t)}$ and the weighting matrix $\mathbf{W}_{i_{k}}^{(t)}$ using (23) (line 14). See details in subsection IV-B. Let us emphasize the following key points about the proposed algorithm for D-CoP in Table II:

- it is not needed to feed back either the weighting matrix $\mathbf{W}_{i_{k}}^{(t-1)}$ or the updated receive filter $\mathbf{R}_{i_{k}}^{(t-1)}$ from the UE to the serving SeNB. They can be reproduced at the SeNB based on the acquired $\mathbf{N}_{i_{k}}^{(t-1)}$ and the transmit filter used in the previous iteration $\left(\mathbf{T}_{i_{k}}^{(t-1)}\right.$ ) which are already known at the SeNB (see line 10). This way, the required feedback links are reduced as compared to [11], where it was assumed that $\mathbf{W}_{i_{k}}$ and $\mathbf{R}_{i_{k}}$ were fed back from each UE to all the SeNBs in the network, while we only need to feed back $\mathbf{C}_{\mathbf{y}_{i}}$ from each UE to the serving SeNB.

- it is not needed to report the updated transmit filter $\mathbf{T}_{i_{k}}^{(t)}$ from the SeNB to the served UE. In practical implementations, the UE estimates the equivalent channel $\mathbf{H}_{k, i_{k}} \mathbf{T}_{i_{k}}^{(t)}$ every time a DL data transmission is carried out, which is enough to compute $\mathbf{R}_{i_{k}}^{(t)}$ in (23) [18].

\section{A. Convergence}

Algorithm in Table II ensures convergence (for $N_{\text {iter }}$ sufficiently large) to a stationary point of $\left(\mathrm{P}_{\mathrm{WMSE}}\right)$ in (18) (which is also a stationary point of ( $\left.\mathrm{P}_{\mathrm{WSR}}\right)$ in (17) [11]) if $\left\{\boldsymbol{\Upsilon}_{k}\right\}$, $\left\{\mathbf{N}_{i_{k}}\right\}$, and $\left\{\mathbf{H}_{k, i_{k}}\right\}, \forall i$, are perfectly acquired at each $k$-th SeNB. Furthermore, the convergence of the algorithm for D$\mathrm{CoP}$ in Table II is proven to be monotonic (i.e. at each iteration $t$, the WSR is increased). 
Theorem 1: If $\boldsymbol{\Upsilon}_{k},\left\{\mathbf{N}_{i_{k}}\right\}$, and $\left\{\mathbf{H}_{k, i_{k}}\right\}, \forall i$, are obtained without errors at each $k$-th SeNB and all SeNBs solve simultaneously its decentralized problem $\left(\mathrm{P}_{\mathrm{WMSE}}^{k}\right)$ in $(20)$, algorithm in Table II converges in a finite number of iterations to a stationary point of problem $\left(\mathrm{P}_{\mathrm{WSR}}\right)$ in (17) and the convergence is monotonic.

Proof: See Appendix B.

The monotonic convergence proof with simultaneous perSeNB optimizations is highly relevant for practical implementation issues, as when only one iteration (or a few) of the algorithm can be performed such proof allows ensuring a performance improvement with parallel and independent per-SeNB optimizations. At the best of our knowledge, this is the first proof of monotonic convergence of an algorithm where all SeNBs update simultaneously their transmit filters to maximize the system WSR in (17) in an interfering multicell multi-user scenario with no information exchange among non-associated terminals. The key point is that we exploit the convexity of the problem with respect to each variable and then, even if we perform parallel and simultaneous perSeNB optimizations, by updating first all $\left\{\mathbf{N}_{i_{k}}\right\}$ and $\left\{\mathbf{R}_{i_{k}}\right\}$ (i.e. line 10 in Table II) and after performing the update of all $\left\{\boldsymbol{\Upsilon}_{k}\right\}$ and $\left\{\mathbf{T}_{i_{k}}\right\}$ (i.e. line 11 in Table II), convergence can be proven. Many proofs of convergence (but not monotonic) with simultaneous per-SeNB optimizations in the literature are based on the insertion of an additional term in the objective function of the problem that allows to linearize the objective function with respect to the optimization variables (see for example [10]), which is not needed in our case.

If errors appear in the estimation of $\Upsilon_{k}, \mathbf{H}_{k, i_{k}}$, or $\mathbf{N}_{i_{k}}$, the update of the DL transmit/receive filters at each $k$-th SeNB can be controlled by slightly reformulating the problem $\left(\mathrm{P}_{\mathrm{WMSE}}^{k}\right)$ in (20) so as to include a proximal point term [16] that controls large deviations due to the estimation errors, similarly as is done in [19]. However, as is pointed out before, the errors in the estimation of $\boldsymbol{\Upsilon}_{k}$ from the UL transmission are very low provided that the scaling cell-wide factor $F$ in (26) is properly designed, and we consistently observe convergence of the proposed algorithm in Table II without including the proximal point term.

\section{B. Complexity}

The complexity of the proposed algorithm is related to the number of iterations $\left(N_{\text {iter }}\right)$, as each iteration requires an UL and a DL transmission and they have to be carried out during the channel coherence time (see Fig. 2). In LTE-A TDD networks, UL and DL transmissions are duplexed according to a set of predefined patterns [20], where in a frame of $10 \mathrm{~ms}$ up to $2 \mathrm{DL}$ and $2 \mathrm{UL}$ transmissions can be duplexed. Hence, 2 iterations of the algorithm could be performed every $10 \mathrm{~ms}$. In a low mobility scenario (mean user speed of $3 \mathrm{Km} / \mathrm{h}$ ), the channel coherence time at the $3.5 \mathrm{GHz}$ band (band devoted to the SCN [6]) results approximately of $25 \mathrm{~ms}$. This way, during the channel coherence time, up to 5 iterations of the algorithm could be implemented. However, in many practical implementations only 1 iteration is allowed, and for that reason in the evaluations $N_{\text {iter }}=1$ is used in many cases (see Section
VI), which allows improving the WSR due to the monotonic convergence proof in Theorem 1.

\section{Practical implementation in 3GPP LTE-A}

In practical implementations a single iteration of the algorithm for D-CoP in Table II would be allowed. In this regard, the proposed D-CoP defines that interference management can be done at the $k$-th SeNB by means of processing the received signal in the UL transmission to get the interferencecost matrix $\hat{\Upsilon}_{k}$ in (28).

In order that each SeNB gets $\hat{\Upsilon}_{k}$ in (28) without affecting the UL data transmission, we can use the already defined sounding reference signals (SRS) in 3GPP LTE-A standard [5] and define an UL pattern-based transmission in which all the active UEs in DL should transmit (see proposals for implementation methods in [21]). SRS sequences should be orthogonal among neighbor UEs in order to properly estimate $\hat{\Upsilon}_{k}$. However, so as to avoid such coordination, pilot sequences composed of random symbols can be used, such that if the sequence length is large enough the orthogonality of the sequences is nearly achieved. In subsection VI-D we evaluate the performance loss due to the use of non-orthogonal UL pilot signals to estimate $\hat{\boldsymbol{\Upsilon}}_{k}$.

The acquisition of $\hat{\Upsilon}_{k}$ in (28) assumes perfect UL-DL propagation channel reciprocity, which requires perfect hardware calibration of the radio-frequency chains in TDD systems [22][23]. Although perfect calibration can be achieved at SeNBs because the variability of surrounding scenario is relatively slow and hardware performances tend to be stable, calibration at UEs may be imperfect due to environmental variations caused by time, power and/or temperature [24]. In this case, the UL and DL propagation channel matrices from the $k$-th SeNB to the $j_{l}$-th UE are related by [22]:

$$
\overleftarrow{\mathbf{H}}_{k, j_{l}}=\mathbf{H}_{k, j_{l}}^{T} \mathbf{C}_{j_{l}}
$$

where $\mathbf{C}_{j_{l}}$ is a diagonal matrix containing calibration errors at the $j_{l}$-th UE. Therefore, calibration errors at UEs may affect the estimation of the interference-cost matrix. In subsection VI-D we evaluate the performance loss due to non-ideal propagation channel reciprocity conditions to estimate $\hat{\boldsymbol{\Upsilon}}_{k}$.

\section{Vi. Evaluations in Small Cell Networks}

The evaluation of the proposed approaches is done on a LTE-Advanced non-co-channel HCNs deployment [6], where macrocells and small cells are not using the same carrier frequency: MeNBs operate at $2 \mathrm{GHz}$ band and SeNBs use the $3.5 \mathrm{GHz}$ band, both with $10 \mathrm{MHz}$ bandwidth conformed of 50 resource blocks (RBs) each. It is used the Small Cell Scenario 2a, following deployment and simulation parameters specified in [6]. The deployment consists of a hexagonal grid with 21 macrocell areas (each covered by a MeNB). One cluster of $\mathrm{SeNBs}$ is deployed per macrocell area, each consisting of 4 or $10 \mathrm{SeNBs} / c l u s t e r$. 60 users are deployed per macrocell area, being $2 / 3$ of them placed inside the cluster and the remaining UEs are uniformly distributed within the macrocell area. $80 \%$ of users are indoor and $20 \%$ of the users are placed outdoor. ITU UMa and ITU UMi models with 3D distance are used 
for path loss and shadowing modeling for MeNB-UE and SeNB-UE links, respectively. For fast fading modeling, the typical urban model is used. Transmit powers are $46 \mathrm{dBm}$, $30 \mathrm{dBm}$ and $23 \mathrm{dBm}$ at MeNB, SeNB and UE, respectively, and antenna gain of $17 \mathrm{dBi}$ at MeNB, $5 \mathrm{dBi}$ at SeNB and $0 \mathrm{dBi}$ at UE. Noise spectral density is $-174 \mathrm{dBm} / \mathrm{Hz}$. The number of antennas is $M_{\mathrm{MeNB}}=2, M_{\mathrm{SeNB}}=2$ and $N_{\mathrm{UE}}=2$ (such that $m_{i_{k}}=2, \forall i, \forall k$, in (5)).

Cell selection at each UE is based on reference-signalreceived-quality (RSRQ) for inter-frequency selection and reference-signal-received-power (RSRP) for intra-frequency selection [6]. After the cell selection we focus on the SCN, i.e. the UEs that have been associated to SeNBs. The number (or percentage) of UEs offloaded to the SCN for each layout configuration is: $68 \%$ for $4 \mathrm{SeNBs} /$ cluster and $75 \%$ for 10 SeNBs/cluster [25] (i.e. 40 and $45 \mathrm{UEs} /$ cluster, respectively).

Two traffic models are implemented:

- Full-load traffic model, where all UEs in the network have packets to be transmitted.

- FTP3 traffic model, with various packet arrival rates $(\lambda$, in packets/s) that lead to different traffic load conditions [6]. In FTP3 traffic model, packets for the same UE arrive according to a Poisson process with arrival rate $\lambda$. The packet size is 0.5 Mbytes.

The active UEs associated to the same SeNB are uniformly distributed among the available resource blocks (RBs) such that intra-cell interference is removed and only inter-cell interference remains. Hence, on each RB each SeNB serves a single UE (i.e. $I_{k}=1, \forall k$, so $I=K$ ). The power available at each SeNB is uniformly distributed among the RBs where the active UEs have been scheduled.

The following techniques are evaluated on each RB (where the system model in Section II applies):

- D-IWA [26]: decentralized procedure where each SeNB designs its transmit filter based on the channel knowledge $\mathbf{H}_{k, i_{k}}$ and acquired $\mathbf{N}_{i_{k}}$ from the served UE, but using $\mathbf{\Upsilon}_{k}=\mathbf{0}$. It is equivalent to performing simultaneous MIMO Iterative Water-filling Algorithms (IWA) in [26].

- D-CoP: decentralized interference management technique detailed in Section IV and Table II to solve problem $\left(\mathrm{P}_{\mathrm{WMSE}}\right)$ in (18), where each SeNB designs its transmit filter based on direct channel knowledge $\mathbf{H}_{k, i_{k}}$, acquired $\mathbf{N}_{i_{k}}$ from its served UE, and an estimation of the interference-cost matrix $\hat{\boldsymbol{\Upsilon}}_{k}$ that is obtained through the UL transmission as in (28).

- $D$-CoP ideal: similar to $D$-CoP, but using the ideal value of $\boldsymbol{\Upsilon}_{k}$ in (19) at each $k$-th SeNB.

- $D$-WMMSE [11]: decentralized procedure presented in [11] to solve problem ( $\left.\mathrm{P}_{\mathrm{WMSE}}\right)$ in (18), where channel matrices from each SeNB to all UEs in the network are known at each $k$-th $\operatorname{SeNB}$ (i.e. $\mathbf{H}_{k, j_{l}}, \forall j, \forall l$ ). Its performance is equal to $C$-WMMSE.

- $C$-WMMSE: centralized interference management procedure to solve problem $\left(\mathrm{P}_{\mathrm{WMSE}}\right)$ in $(18)$, in which case a central unit processor is assumed to collect channel matrices from all SeNBs to all UEs (i.e. $\mathbf{H}_{k, j_{l}}, \forall k, \forall j, \forall l$ ).

For all techniques, two optimization strategies are evaluated:
- minWMSE: solves ( $\left.\mathrm{P}_{\mathrm{WMSE}}\right)$ in (18) with $\mathbf{W}_{i_{k}}=\mathbf{I}, \forall i, \forall k$,

- maxWSR: solves ( $\mathrm{P}_{\mathrm{WMSE}}$ ) in (18) with $\mathbf{W}_{i_{k}}$ properly optimized so as to achieve the maximum WSR. $\mu_{i_{k}}=1$, $\forall i, \forall k$, is used.

The number of iterations is set to $N_{\text {iter }}=1$, except for the convergence evaluations in subsection VI-A, and in some cases $N_{\text {iter }}=10$ is used for comparison purposes.

The performance indicator is user packet throughput (UPT) measured in Mbits/s and defined as the amount of data over the time needed to download data, without including the packet waiting time in the buffer. Maximum modulation and coding schemes from LTE-A and $3 \mathrm{~dB}$ of coding losses are used. For some purposes we evaluate the per-user achievable rate on a specific RB, according to (12) and measured in bits/s/Hz.

\section{A. Convergence}

The monotonic convergence of the proposed $\mathrm{D}-\mathrm{CoP}$ is demonstrated on a specific RB without considering channel estimation errors, for the configuration of 4 and 10 SeNBs/cluster, when using the full-load traffic model. Fig. 3.a shows the convergence in terms of per-user MSE for minWMSE strategy (let us recall that $m_{i_{k}}=2$ is used for all UEs in the problem set up). Fig. 3.b displays the convergence in terms of per-user achievable rate (in bits/s/Hz) for maxWSR strategy. In both figures, the solution achieved with the centralized procedure is displayed with a dotted line.

It can be observed that D-CoP ideal has a monotonic convergence, while the proposed $\mathrm{D}-\mathrm{CoP}$ based on propagation channel reciprocity also converges due to the fact that estimation errors in the acquisition of $\boldsymbol{\Upsilon}_{k}$ from UL are not relevant (see justification in subsection IV-D). The convergence speed of D-CoP is faster than D-WMMSE [11] because with the proposed approach an alternate optimization can be performed at each SeNB on each iteration of the algorithm, hence achieving a faster convergence. C-WMMSE and D-WMMSE [11] approaches have the same performance result when a large number of iterations is used, because the equations employed to achieve the solution are equivalent.

The proposed D-CoP achieves a system performance very similar to C-WMMSE and D-WMMSE [11] approaches, but with much less overhead and complexity (in fact, the complexity of D-CoP is comparable to D-IWA [26]). As the problem is non-convex with respect to all variables, all the interference management approaches only achieve a local minimum, so in some channel realizations they lead to the same solution (as in the minWMSE case in Fig. 3.a) while in others the attained locally optimal solution is different (as in the maxWSR case in Fig. 3.b). As compared to the baseline D-IWA scheme [26], the reduction in the per-user MSE and the increase in the per-user achievable rate are larger in the layout of $10 \mathrm{SeNB}$ /cluster, as more interference is present in the SCN. Furthermore, it is important to recall that when performing only $N_{\text {iter }}=1$ iteration, the proposed D-CoP already outperforms the baseline D-IWA [26] scheme, which might not happen with D-WMMSE [11] because the decentralized optimizations at SeNBs with D-CoP allow converging faster to a stable point. 


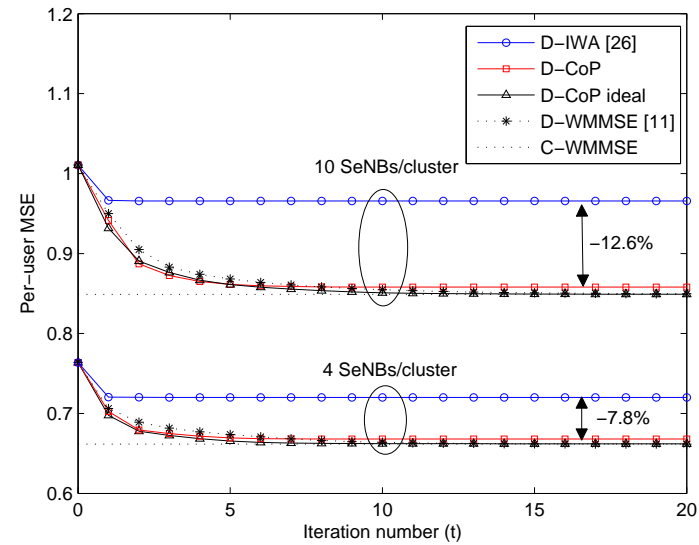

(a) Per-user MSE, for minWMSE strategy

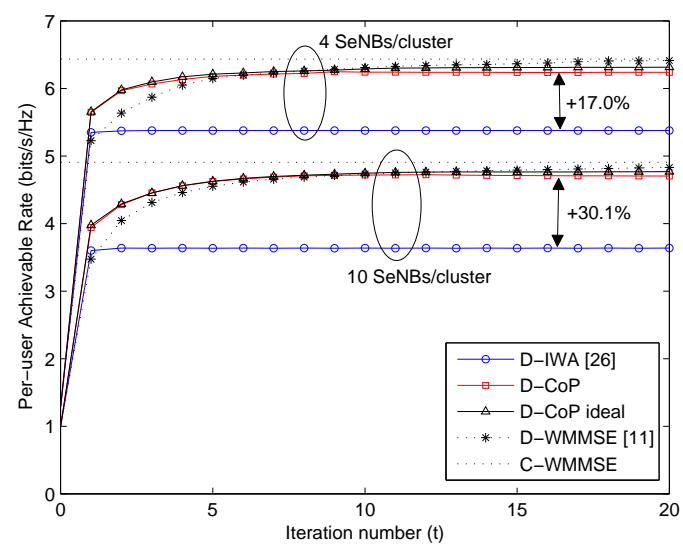

(b) Per-user achievable rate, for maxWSR strategy

Fig. 3: Convergence in terms of per-user MSE for $\min W M S E$ strategy (left-figure) and per-user achievable rate (in bits/s/Hz) for maxWSR strategy (right-figure) on a specific resource block, for the layout configuration of 4 and $10 \mathrm{SeNBs} / \mathrm{cluster}$

\section{B. Evaluations under perfect CSI conditions}

Fig. 4 displays the 5\%-tile UPT vs. the mean UPT for the configuration of 4 and $10 \mathrm{SeNBs} /$ cluster, when using the fullload traffic model and minWMSE or maxWSR strategy. The performance of D-CoP and D-WMMSE [11] are included for $N_{\text {iter }}=1$ and $N_{\text {iter }}=10$. On the one side, the minWMSE strategy allows improving specially the 5\%-tile UPT, and $N_{\text {iter }}=1$ iteration is shown to be enough with D-CoP to get close to the performance given by the ideal centralized approach. On the other side, the maxWSR strategy provides larger gains in the mean UPT, but as the number of iterations increases the 5\%-tile UPT is degraded. In this case the number of iterations is relevant in some cases, as with 10 iterations the mean UPT is enlarged specially for the layout of 10 SeNBs/cluster where more interference is present. However, the larger mean UPT gain with D-CoP comes in the first iteration, as is shown in Fig. 3.b, because the decentralized optimization proposed at SeNBs with D-CoP allows a faster convergence (and it does not happen with D-WMMSE [11]).

Fig. 5 shows the 5\%-tile UPT vs. the mean UPT for different values of the packet arrival rate $(\lambda=0.5,1,1.5,2)$ of the Poisson distribution used in FTP3 traffic model, for the configuration of 4 and $10 \mathrm{SeNBs} /$ cluster. maxWSR optimization

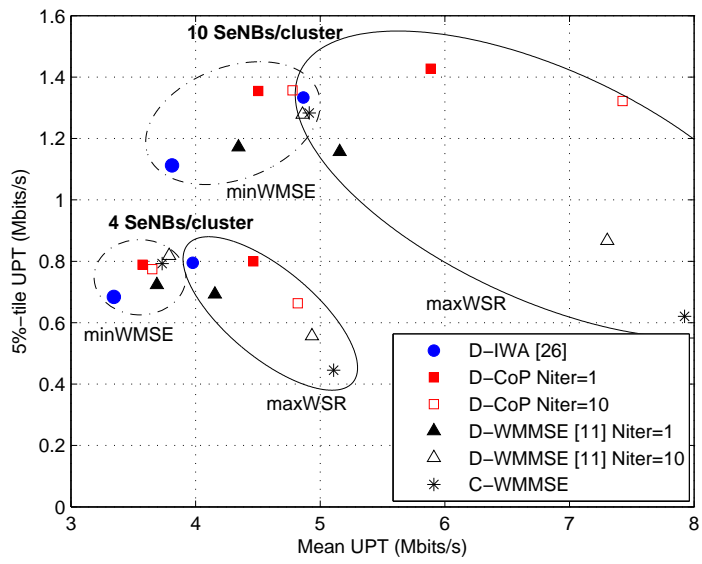

Fig. 4: 5\%-tile UPT (Mbits/s) vs. mean UPT (Mbits/s) in the full-load traffic model, for the layout configuration of 4 and $10 \mathrm{SeNB} /$ cluster, when using maxWSR and minWMSE optimization strategies. $N_{\text {iter }}=1$ and $N_{\text {iter }}=10$ are displayed.

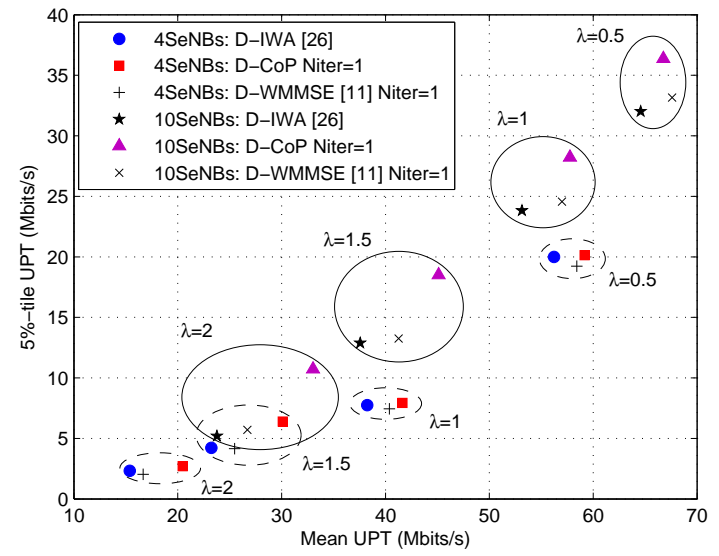

Fig. 5: 5\%-tile UPT (Mbits/s) vs. mean UPT (Mbits/s) for different packet arrival rates ( $\lambda$ in packets/s) in the FTP3 traffic model, for the layout configuration of 4 and 10 SeNBs/cluster, when using maxWSR optimization strategy. $N_{\text {iter }}=1$ is used.

strategy and $N_{\text {iter }}=1$ are used. D-CoP is compared to DIWA [26] that requires a similar complexity, and also to DWMMSE [11] that requires more channels to be estimated and more feedback links. As expected, both the 5\%-tile UPT and the mean UPT decrease as the offered load (i.e. $\lambda$ ) increases for all simulation cases. The UPT gains of D-CoP are larger for the $10 \mathrm{SeNBs} / \mathrm{cluster}$ deployment and they are also larger when $\lambda$ increases, as more interference is present in the SCN either due to a denser deployment of SeNBs or due to a traffic load increase. The UPT gains provided with $N_{\text {iter }}=1$ iteration are larger for D-CoP than for D-WMMSE [11], as was already observed in Fig. 3.b.

In general, both in 5\%-tile and mean UPT gains are obtained when using the maxWSR strategy due to the fact that each SeNB has $50 \mathrm{RBs}$ available where the served UEs are uniformly distributed. Then, as each user is allocated to multiple RBs, the probability of being in outage in all the assigned RBs is very low and, even if we maximize the system WSR on each RB, we get an increase in the 5\%-tile UPT. This effect is more remarkable in the $10 \mathrm{SeNB} /$ cluster deployment and for the FTP3 traffic model, where more resources are available.

In conclusion, a significant UPT performance gain of the 


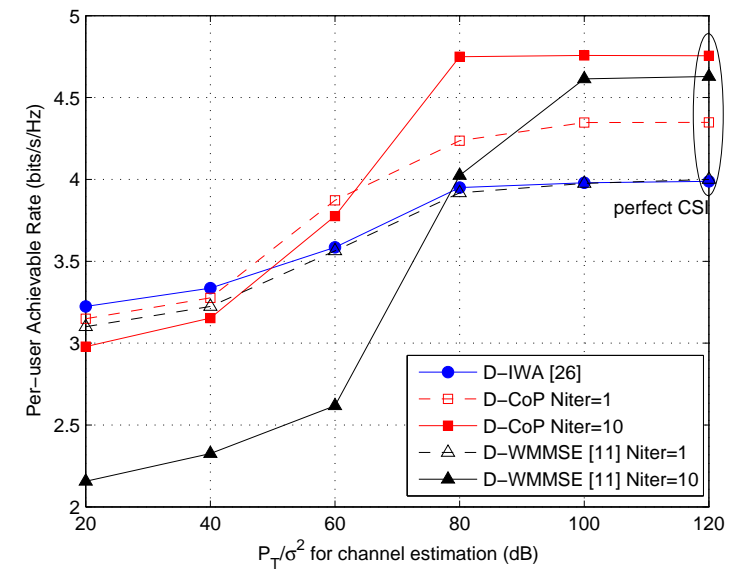

Fig. 6: Per-user achievable rate on a specific resource block (bits/s/Hz) vs. $P_{T} / \sigma_{v}^{2}$ used for channel estimation $(\mathrm{dB})$ in the full-load traffic model, for the layout configuration of $4 \mathrm{SeNBs} / \mathrm{cluster}$, when using $\operatorname{maxWSR}$ optimization strategy. $N_{\text {iter }}=1$ and $N_{\text {iter }}=10$ are used.

proposed D-CoP and $N_{\text {iter }}=1$ with respect to the baseline D-IWA [26] scheme and with respect to D-WMMSE [11] is observed in all layout configurations and traffic simulation conditions. The relative gains are larger for medium-to-high traffic loads (up to a certain limit) and for denser deployments of SeNBs, due to the fact that D-CoP allows a full reuse of sub-bands and sub-frames while preemptively managing interference at UEs and, especially, at cell-edge UEs with a low complexity implementation. So it can be concluded that $\mathrm{D}-\mathrm{CoP}$ is a suitable approach for interference limited scenarios and dense networks. It is important to recall that large UPT gains are obtained even if only one iteration of the proposed $\mathrm{D}-\mathrm{CoP}$ is performed and that no additional overhead is needed for D-CoP if we use an UL pilot-based transmission to get the interference-cost matrix, which makes the approach amenable for practical system implementations.

\section{Evaluations under imperfect CSI conditions}

As is shown in Table $\mathrm{I}$ and subsection IV-A2, robust precoders are designed according to the adopted interference management procedure (D-WMMSE [11] or D-CoP). Fig. 6 displays the per-user achievable rate on a specific RB (in bits/s/Hz) vs. $P_{T} / \sigma_{v}^{2}$ used during the training phase (see definition in (4)) for the configuration of $4 \mathrm{SeNBs} /$ cluster, when using the full-load traffic model. Let us recall that the results with $P_{T} / \sigma_{v}^{2}=120 \mathrm{~dB}$ are nearly equal to the results with perfect CSI. For low values of $P_{T} / \sigma_{v}^{2}$, the performance loss due to imperfect CSI is larger for $N_{\text {iter }}=10$ than for $N_{\text {iter }}=1$ due to the propagation of errors in the iterative algorithms for D-WMMSE and D-CoP.

It can be observed that as $P_{T} / \sigma_{v}^{2}$ is reduced, the performance of the D-WMMSE [11] decreases drastically with $N_{\text {iter }}=10$ due to the errors in the estimation of interfering channels (see $\boldsymbol{\Phi}_{k}$ in (30)). However, the proposed D-CoP and the baseline D-IWA [26] schemes are shown to be more robust to imperfect CSI because they only involve estimation errors in the direct channel matrix towards the serving SeNB. This shows that UPT gains can be obtained with the proposed DCoP technique even if the channels are estimated with a low

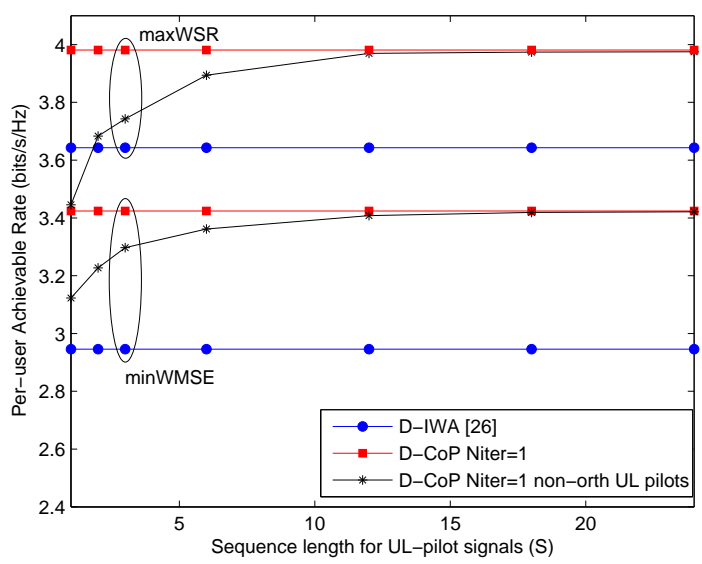

Fig. 7: Per-user achievable rate (bits/s/Hz) on a specific resource block vs. UL pilot sequence length $(S)$ in the full-load traffic model, for the layout configuration of 10

SeNBs/cluster, when using $\min W M S E$ or $\max W S R$ strategy. $N_{\text {iter }}=1$ is used.

SNR, which is not the case with conventional transmit coordination techniques that require estimation of all the channel matrices. Let us recall that in case C-WMMSE approach was used, then the performance would be even worse than DWMMSE [11] because $\mathbf{N}_{i_{k}}$ in (22) would also include the estimation errors of the interfering channel matrices.

\section{Evaluations under real impairments to acquire $\hat{\Upsilon}_{k}$}

In this subsection we evaluate the effect of real impairments on the the acquisition of $\hat{\Upsilon}_{k}$ in (28) for the proposed D-CoP, as introduced and discussed in subsection $\mathrm{V}-\mathrm{C}$.

First, we consider the case in which non-orthogonal UL pilot sequences are used to estimate $\hat{\Upsilon}_{k}$. Sequences composed of $\{+1,-1\}$ random symbols are used, with a length equal to $S$. Fig. 7 shows the per-user achievable rate (in bits/s/Hz) on a specific RB vs. $S$ when using the full-load traffic model for the configuration of $10 \mathrm{SeNBs} /$ cluster. $N_{\text {iter }}=1$ is used for D-CoP. It can be observed that if non-orthogonal random sequences are used to get $\hat{\Upsilon}_{k}$, even with a low number of symbols $(S)$, the performance is near to the one with orthogonal sequences among all UEs. Let us recall that in practical LTE-A implementations $S=12$ can be used within a $\mathrm{RB}$, such that the effect of non-orthogonal UL pilot sequences would be negligible.

Second, we evaluate the impact of non-ideal channel reciprocity conditions to acquire $\hat{\Upsilon}_{k}$. The diagonal entries of the calibration error matrix $\mathbf{C}_{j_{l}}$ in (31) are modeled by: $\left(1+a_{j_{l}}\right) e^{j \phi_{j_{l}}}$, being $a_{j_{l}} \sim \mathcal{T} \mathcal{N}\left(0, \sigma_{a}^{2},-1,1\right)$ and $\phi_{j_{l}} \sim$ $\mathcal{T N}\left(0, \sigma_{\phi}^{2},-\pi / 6, \pi / 6\right)$ random variables with a truncated normal distribution that include the calibration error (CE) in amplitude and phase, respectively [22]. The variance for amplitude and phase CE at UEs $\left(\sigma_{a}^{2}\right.$ and $\left.\sigma_{\phi}^{2}\right)$ are assumed to be equal for all UEs, and are taken as an input for the simulations. Fig. 8 shows the per-user achievable rate (in bits/s/Hz) on a specific RB vs. $\sigma^{2}$ (which either refers to the variance of the amplitude $\mathrm{CE}, \sigma_{a}^{2}$, and/or to the variance of the phase $\mathrm{CE}, \sigma_{\phi}^{2}$, as is indicated in the legend). We have used the configuration of $10 \mathrm{SeNBs} /$ cluster, the full-load traffic model and maxWSR strategy. The performance of D-CoP is included for $N_{\text {iter }}=1$ and $N_{\text {iter }}=10$. It can be observed 


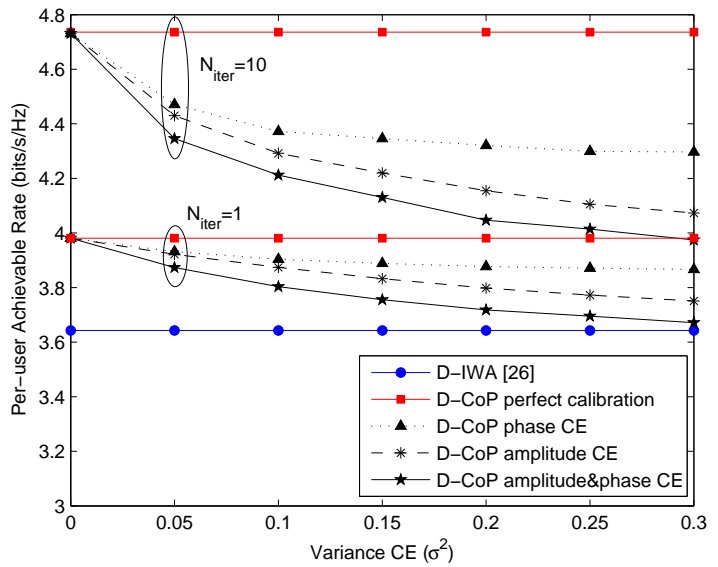

Fig. 8: Per-user achievable rate (bits/s/Hz) on a specific resource block vs. $\sigma^{2}$ (either referring to the variance in phase and/or amplitude CE) in the full-load traffic model, for the layout configuration of $10 \mathrm{SeNBs/cluster,} \mathrm{when} \mathrm{using} \mathrm{maxWSR} \mathrm{strategy.}$ $N_{\text {iter }}=1$ and $N_{\text {iter }}=10$ are displayed.

that the relative performance loss as compared to the perfect calibration case is larger for $N_{\text {iter }}=10$ than $N_{\text {iter }}=1$, due to the propagation of errors in the iterative algorithm: at each iteration, the interference-cost matrix is acquired with errors which impacts on the transmit/receive filters design and, on its turn, on the interference-cost matrix. As more iterations are done, higher impact of these errors on performance is observed. The impact of amplitude CE is a bit more harmful than phase CE, but in MIMO systems phase calibration at UEs is also important. However, even with high variances of the CE (note that, for example [23] considers values of $\sigma_{a}^{2}=\sigma_{\phi}^{2}=0.01$, while we are displaying values up to $\sigma_{a}^{2}=\sigma_{\phi}^{2}=0.3$ ), the performance of D-CoP outperforms the baseline D-IWA [26] scheme.

\section{CONCLUSIONS}

This article presents a decentralized coordinated precoding (D-CoP) design for weighted sum-rate maximization in dense MIMO TDD SCNs. Precoding decisions are done at each SeNB based on the knowledge of the propagation channel towards its served UEs, the acquired DL interference, and the received signal in the UL. The received signal in the UL allows to estimate the interference-cost matrix, which informs about how the SeNB is interfering to unintended UEs. This way, it is not needed to estimate the interfering channels either at UEs or at SeNBs, no backhaul traffic between SeNBs is required, and minimal reporting of information from the UE to the serving SeNB is needed. Then, an iterative algorithm for $\mathrm{D}-\mathrm{CoP}$ is presented, which subsumes the acquisition of the required parameters and the simultaneous per-SeNB optimizations. Monotonic convergence of the algorithm is demonstrated when all SeNBs update its transmit precoders in parallel. Both the monotonic convergence and the parallel optimizations are highly relevant for practical implementation issues. Furthermore, a robust D-CoP is presented to tackle imperfect CSI conditions, and the impact of non-ideal propagation channel reciprocity is analyzed. Significant gains in terms of user packet throughput, and specially 5\%-tile UPT, are observed in 3GPP LTE-A SCNs for all layout configurations and all traffic simulation conditions, as compared to a baseline scheme with comparable complexity. The UPT gains are comparable to well-known centralized and decentralized interference management techniques, which involve much more complexity. The use of only one iteration of the algorithm allows improving the UPT and the relative gains are larger for mediumto-high traffic loads and for denser deployments of SeNBs, which demonstrates the suitable applicability of the proposed D-CoP in interference-limited scenarios. Further, the proposed scheme is shown through simulations to be robust to imperfect CSI conditions as well as real impairments in the estimation of the interference-cost matrix, like the use of non-orthogonal UL pilot sequences at UEs and non-ideal propagation channel reciprocity conditions.

\section{APPENDIX A PROOF OF PROPOSITION 1}

On the one hand, the Lagrangian function $(\mathcal{L})$ of the problem $\left(\mathrm{P}_{\mathrm{WMSE}}\right)$ in (18) is [17]:

$$
\begin{aligned}
\mathcal{L} & =\sum_{k=1}^{K} \sum_{i=1}^{I_{k}}\left(\operatorname{Tr}\left(\mathbf{W}_{i_{k}} \mathbf{E}_{i_{k}}\left(\mathbf{R}_{i_{k}},\left\{\mathbf{T}_{i_{k}}\right\}\right)\right)-\mu_{i_{k}} \log _{2}\left|\mu_{i_{k}}^{-1} \mathbf{W}_{i_{k}}\right|\right) \\
& +\sum_{k=1}^{K} \lambda_{k}\left(\sum_{i=1}^{I_{k}} \operatorname{Tr}\left(\mathbf{T}_{i_{k}} \mathbf{T}_{i_{k}}^{H}\right)-P_{k}^{\mathrm{SeNB}}\right)
\end{aligned}
$$

being $\mathbf{E}_{i_{k}}\left(\mathbf{R}_{i_{k}},\left\{\mathbf{T}_{i_{k}}\right\}\right)$ defined in (18).

On the other hand, the Lagrangian function $\left(\mathcal{L}_{k}\right)$ of the decentralized problem ( $\mathrm{P}_{\mathrm{WMSE}}^{k}$ ) in (20) for fixed $\boldsymbol{\Upsilon}_{k}$ and $\mathbf{N}_{i_{k}}$, $\forall i$ is:

$$
\begin{aligned}
\mathcal{L}_{k}= & \sum_{i=1}^{I_{k}}\left(\operatorname{Tr}\left(\mathbf{W}_{i_{k}} \mathbf{E}_{i_{k}}\left(\mathbf{R}_{i_{k}},\left\{\mathbf{T}_{i_{k}}\right\}, \mathbf{N}_{i_{k}}\right)\right)-\mu_{i_{k}} \log _{2}\left|\mu_{i_{k}}^{-1} \mathbf{W}_{i_{k}}\right|\right. \\
& \left.+\operatorname{Tr}\left(\boldsymbol{\Upsilon}_{k} \mathbf{T}_{i_{k}} \mathbf{T}_{i_{k}}^{H}\right)\right)+\lambda_{k}\left(\sum_{i=1}^{I_{k}} \operatorname{Tr}\left(\mathbf{T}_{i_{k}} \mathbf{T}_{i_{k}}^{H}\right)-P_{k}^{\mathrm{SeNB}}\right),(33)
\end{aligned}
$$

being $\mathbf{E}_{i_{k}}\left(\mathbf{R}_{i_{k}},\left\{\mathbf{T}_{i_{k}}\right\}, \mathbf{N}_{i_{k}}\right)$ the MSE-matrix in (18) assuming that $\mathbf{N}_{i_{k}}$ is fixed. Note that the Lagrangian function $\mathcal{L}_{k}$ and other $\mathcal{L}_{l}, \forall l \neq k$, are not coupled through any variable as the matrices containing inter-cell interference have been fixed (i.e. $\boldsymbol{\Upsilon}_{k}$ and $\mathbf{N}_{i_{k}}, \forall i$ ).

It is easy to check that the derivative of the Lagrangian functions in (32) and (33) with respect to $\mathbf{W}_{i_{k}}$ coincide, $\forall i$, $\forall k$, when $\boldsymbol{\Upsilon}_{k}$ is fixed. Further, it is also easy to check that the derivatives with respect to $\mathbf{R}_{i_{k}}$ are equal when $\boldsymbol{\Upsilon}_{k}$ is fixed, $\forall i$, $\forall k$, as $\mathbf{R}_{i_{k}}$ is only included in $\mathbf{E}_{i_{k}}$. So, let us focus on checking that the gradients coincide when we derive with respect to $\mathbf{T}_{i_{k}}$.

The derivative of $\mathcal{L}$ in (32) with respect to $\mathbf{T}_{i_{k}}$ is:

$$
\begin{aligned}
\frac{d}{d \mathbf{T}_{i_{k}}} \mathcal{L}= & \left(\sum_{j=1}^{I_{k}} \mathbf{H}_{k, j_{k}}^{H} \mathbf{R}_{j_{k}} \mathbf{W}_{j_{k}} \mathbf{R}_{j_{k}}^{H} \mathbf{H}_{k, j_{k}}\right) \mathbf{T}_{i_{k}}-\mathbf{H}_{k, i_{k}}^{H} \mathbf{R}_{i_{k}} \mathbf{W}_{i_{k}} \\
& +\left(\sum_{\substack{l=1 \\
l \neq k}}^{K} \sum_{j=1}^{I_{l}} \mathbf{H}_{k, j_{l}}^{H} \mathbf{R}_{j_{l}} \mathbf{W}_{j_{l}} \mathbf{R}_{j_{l}}^{H} \mathbf{H}_{k, j_{l}}\right) \mathbf{T}_{i_{k}}+\lambda_{k} \mathbf{T}_{i_{k}},(34)
\end{aligned}
$$

where we have taken into account that $\mathbf{T}_{i_{k}}$ affects not only $\mathbf{E}_{i_{k}}$, but also the MSE-matrices of the remaining UEs (i.e. $\mathbf{E}_{j_{l}}, \forall l, \forall j$ ). 
The derivative of $\mathcal{L}_{k}$ in (33) with respect to $\mathbf{T}_{i_{k}}$, when $\boldsymbol{\Upsilon}_{k}$ and $\mathbf{N}_{i_{k}}, \forall i$, are fixed, is:

$$
\begin{aligned}
\frac{d}{d \mathbf{T}_{i_{k}}} \mathcal{L}_{k}= & \left(\sum_{j=1}^{I_{k}} \mathbf{H}_{k, j_{k}}^{H} \mathbf{R}_{j_{k}} \mathbf{W}_{j_{k}} \mathbf{R}_{j_{k}}^{H} \mathbf{H}_{k, j_{k}}\right) \mathbf{T}_{i_{k}}-\mathbf{H}_{k, i_{k}}^{H} \mathbf{R}_{i_{k}} \mathbf{W}_{i_{k}} \\
& +\mathbf{\Upsilon}_{k} \mathbf{T}_{i_{k}}+\lambda_{k} \mathbf{T}_{i_{k}},
\end{aligned}
$$

where it is important to recall that in this case $\mathbf{T}_{i_{k}}$ only affects the MSE-matrices of the UEs served by the $k$ th $\operatorname{SeNB}$ (i.e. $\mathbf{E}_{j_{k}}, \forall j$ ), as $\mathbf{N}_{i_{k}}, \forall i$, have been fixed. Therefore, the gradients in (34) and in (35) are equal if $\mathbf{\Upsilon}_{k}=\sum_{l=1, l \neq k}^{K} \sum_{j=1}^{I_{l}} \mathbf{H}_{k, j_{l}}^{H} \mathbf{R}_{j_{l}} \mathbf{W}_{j_{l}} \mathbf{R}_{j_{l}}^{H} \mathbf{H}_{k, j_{l}}$ is set.

\section{APPENDIX B}

\section{PROOF OF THEOREM 1}

Convergence of the algorithm in Table II into a stationary point of problem $\left(\mathrm{P}_{\mathrm{WSR}}\right)$ in (17) can be proved by grace of monotonic convergence of the objective function of problem $\left(\mathrm{P}_{\mathrm{WMSE}}\right)$ in (18) [11]. Let us express the objective function of the problem $\left(\mathrm{P}_{\mathrm{WMSE}}\right)$ in $(18)$ as follows:

$$
\begin{aligned}
& f_{o}\left(\left\{\mathbf{T}_{i_{k}}\right\},\left\{\mathbf{R}_{i_{k}}\right\},\left\{\mathbf{W}_{i_{k}}\right\}\right)= \\
& \sum_{k=1}^{K} \sum_{i=1}^{I_{k}}\left(\operatorname{Tr}\left(\mathbf{W}_{i_{k}} \mathbf{E}_{i_{k}}\left(\mathbf{R}_{i_{k}},\left\{\mathbf{T}_{i_{k}}\right\}\right)\right)-\chi_{i_{k}}\right),
\end{aligned}
$$

where $\chi_{i_{k}}=\mu_{i_{k}} \log _{2}\left|\mu_{i_{k}}^{-1} \mathbf{W}_{i_{k}}\right| . f_{o}($.$) in (36) is a convex$ function with respect to each set of optimization variables separately (i.e. $\left\{\mathbf{T}_{i_{k}}\right\},\left\{\mathbf{R}_{i_{k}}\right\}$ and $\left\{\mathbf{W}_{i_{k}}\right\}$ ). In particular, for fixed weighting matrices, $f_{o}($.$) is convex with respect to$ transmit (receive) filters for a set of given receive (transmit) filters. In our proposed D-CoP procedure, we need to demonstrate a reduction of $f_{o}($.$) in (36) whenever any of the three$ sets of optimization variables is updated. In particular, when performing lines 11, 12, and 14 in Table II. These updates are summarized in four steps:

- Step 1 (line 11) (for all SeNBs): Update of the transmit filters $\left\{\mathbf{T}_{i_{k}}\right\}, \forall i$, (denoted by $\left\{\mathbf{T}_{i_{k}}^{\text {aux }}\right\}, \forall i$ ) based on the acquired interference-cost matrix $\boldsymbol{\Upsilon}_{k}$ at each SeNB.

- Step 2 (line 12) (for all SeNBs): Alternate optimization of the receive filters $\mathbf{R}_{i_{k}}$, weighting matrices $\mathbf{W}_{i_{k}}$, and transmit filters $\mathbf{T}_{i_{k}}, \forall i$, at each SeNB for a fixed $\left\{\mathbf{N}_{i_{k}}\right\}$, $\forall i$, and $\mathbf{\Upsilon}_{k}$.

- Step 3 (line 14) (for all UEs): Update of the receive filter $\mathbf{R}_{i_{k}}$ based on the actual covariance matrix of the received inter-cell interference plus noise per UE $\mathbf{N}_{i_{k}}$.

- Step 4 (line 14) (for all UEs): Update of the weighting matrix per UE $\mathbf{W}_{i_{k}}$.

In the following proof it is assumed that $\boldsymbol{\Upsilon}_{k},\left\{\mathbf{N}_{i_{k}}\right\}$, and $\left\{\mathbf{H}_{k, i_{k}}\right\}, \forall i$, are acquired without errors at each $k$-th SeNB (so we focus on the perfect CSI case in subsection IV-A1).

When solving the optimization problem at each SeNB, DL transmit filters are designed at each $k$-th SeNB following the expressions in (21) for fixed $\left\{\mathbf{N}_{i_{k}}\right\}, \forall i$, in (9) and $\boldsymbol{\Upsilon}_{k}$ in (19).
As a consequence, $f_{o}($.$) in (36) can be written as:$

$$
\begin{aligned}
& f_{o}\left(\left\{\mathbf{T}_{i_{k}}\left(\mathbf{\Upsilon}_{k}\right)\right\},\left\{\mathbf{R}_{i_{k}}\left(\mathbf{N}_{i_{k}}\right)\right\},\left\{\mathbf{W}_{i_{k}}\right\}\right)= \\
& \sum_{k=1}^{K} \sum_{i=1}^{I_{k}}\left(\operatorname{Tr}\left(\mathbf{W}_{i_{k}} \mathbf{E}_{i_{k}}\left(\mathbf{R}_{i_{k}}\left(\mathbf{N}_{i_{k}}\right),\left\{\mathbf{T}_{i_{k}}\left(\mathbf{\Upsilon}_{k}\right)\right\}\right)\right)-\chi_{i_{k}}\right) .
\end{aligned}
$$

The key point in this expression is that, for fixed weighting matrices $\left\{\mathbf{W}_{i_{k}}\right\}$, each transmit filter $\mathbf{T}_{i_{k}}$ depends on the receive filters $\left\{\mathbf{R}_{i_{k}}\right\}$ and on the interference-cost matrix $\boldsymbol{\Upsilon}_{k}$ that only depends on receive filters $\left\{\mathbf{R}_{i_{k}}\right\}$. Similarly, for fixed weighting matrices $\left\{\mathbf{W}_{i_{k}}\right\}$, each receive filter $\mathbf{R}_{i_{k}}$ depends on the transmit filter $\left\{\mathbf{T}_{i_{k}}\right\}$ and on the covariance matrix of the received inter-cell interference plus noise $\mathbf{N}_{i_{k}}$ that only depends on transmit filters $\left\{\mathbf{T}_{i_{k}}\right\}$. Now we are ready to prove convergence on each step of the algorithm in Table II. In the sequel, $t$ denotes the iteration number.

- Proof of objective function reduction in Step 1: Step 1 consists on updating $\boldsymbol{\Upsilon}_{k}^{(t)}$ and $\mathbf{T}_{i_{k}}^{\text {aux }}, \forall i, \forall k$. If we use the fact that for the set of fixed $\left\{\mathbf{R}_{i_{k}}^{(t-1)}\right\}$ and $\left\{\mathbf{W}_{i_{k}}^{(t-1)}\right\}$, since $f_{0}($.$) is$ convex on $\left\{\mathbf{T}_{i_{k}}\right\}$, we can update sequentially: $i$ ) all $\left\{\mathbf{\Upsilon}_{k}^{(t)}\right\}$,ii) all $\left\{\mathbf{T}_{i_{k}}^{\text {aux }}\right\}$ following (21), and then convergence is guaranteed because transmit filters given by (21) are the optimum for a given set of $\left\{\mathbf{R}_{i_{k}}^{(t-1)}\right\}$ and $\left\{\mathbf{W}_{i_{k}}^{(t-1)}\right\}$ if $\left\{\mathbf{\Upsilon}_{k}^{(t)}\right\}$ is computed from the same set of $\left\{\mathbf{R}_{i_{k}}^{(t-1)}\right\}$ and $\left\{\mathbf{W}_{i_{k}}^{(t-1)}\right\}$. In other words:

$$
\begin{aligned}
& f_{o}\left(\left\{\mathbf{T}_{i_{k}}^{(t-1)}\right\},\left\{\mathbf{R}_{i_{k}}^{(t-1)}\left(\mathbf{N}_{i_{k}}^{(t-1)}\right)\right\},\left\{\mathbf{W}_{i_{k}}^{(t-1)}\right\}\right) \geq \\
& f_{o}\left(\left\{\mathbf{T}_{i_{k}}^{\text {aux }}\left(\mathbf{\Upsilon}_{k}^{(t)}\right)\right\},\left\{\mathbf{R}_{i_{k}}^{(t-1)}\left(\mathbf{N}_{i_{k}}^{(t-1)}\right)\right\},\left\{\mathbf{W}_{i_{k}}^{(t-1)}\right\}\right) .
\end{aligned}
$$

- Proof of objective function reduction in Step 2: Precoding design at each SeNB follows a block coordinate descent method [16] with alternate optimization between receive filters, weighting matrices, and transmit filters in (21). Therefore, an objective reduction for a fixed $\left\{\mathbf{N}_{i_{k}}^{(t-1)}\right\}, \forall i$, and $\Upsilon_{k}^{(t)}$ at each $k$-th SeNB is guaranteed if the alternate optimization starts from $\left\{\mathbf{T}_{i_{k}}^{\text {aux }}\right\}$. The variables as a result of the alternate optimization are denoted by: $\left\{\mathbf{T}_{i_{k}}^{(t)}\right\},\left\{\mathbf{R}_{i_{k}}^{\text {aux }}\right\},\left\{\mathbf{W}_{i_{k}}^{\text {aux }}\right\}$. As each subproblem converges, convergence of the sum is guaranteed for a fixed $\left\{\mathbf{N}_{i_{k}}^{(t-1)}\right\}$ and $\mathbf{\Upsilon}_{k}^{(t)}, \forall i, \forall k$ :

$$
\begin{aligned}
& f_{o}\left(\left\{\mathbf{T}_{i_{k}}^{\text {aux }}\left(\mathbf{\Upsilon}_{k}^{(t)}\right)\right\},\left\{\mathbf{R}_{i_{k}}^{(t-1)}\left(\mathbf{N}_{i_{k}}^{(t-1)}\right)\right\},\left\{\mathbf{W}_{i_{k}}^{(t-1)}\right\}\right) \geq \\
& f_{o}\left(\left\{\mathbf{T}_{i_{k}}^{(t)}\left(\mathbf{\Upsilon}_{k}^{(t)}\right)\right\},\left\{\mathbf{R}_{i_{k}}^{\text {aux }}\left(\mathbf{N}_{i_{k}}^{(t-1)}\right)\right\},\left\{\mathbf{W}_{i_{k}}^{\text {aux }}\right\}\right) .
\end{aligned}
$$

- Proof of objective function reduction in Step 3: Step 3 consists on updating $\mathbf{N}_{i_{k}}^{(t)}$ and $\mathbf{R}_{i_{k}}^{(t)}, \forall i, \forall k$. As $f_{0}($.$) is convex$ on $\left\{\mathbf{R}_{i_{k}}\right\}$ for a set of fixed $\left\{\mathbf{T}_{i_{k}}^{(t)}\right\}$ and $\left\{\mathbf{W}_{i_{k}}^{\text {aux }}\right\}$, we can update sequentially: i) all $\left\{\mathbf{N}_{i_{k}}^{(t)}\right\}$,ii) all $\left\{\mathbf{R}_{i_{k}}^{(t)}\right\}$ following (23), and thereby convergence is guaranteed since receivers given by (23) are the optimum for a given set of $\left\{\mathbf{T}_{i_{k}}^{(t)}\right\}$ if the used $\left\{\mathbf{N}_{i_{k}}^{(t)}\right\}$ is computed from the same set of transmit filters $\left\{\mathbf{T}_{i_{k}}^{(t)}\right\}$. This way:

$$
\begin{aligned}
& f_{o}\left(\left\{\mathbf{T}_{i_{k}}^{(t)}\left(\mathbf{\Upsilon}_{k}^{(t)}\right)\right\},\left\{\mathbf{R}_{i_{k}}^{\text {aux }}\left(\mathbf{N}_{i_{k}}^{(t-1)}\right)\right\},\left\{\mathbf{W}_{i_{k}}^{\text {aux }}\right\}\right) \geq \\
& f_{o}\left(\left\{\mathbf{T}_{i_{k}}^{(t)}\left(\mathbf{\Upsilon}_{k}^{(t)}\right)\right\},\left\{\mathbf{R}_{i_{k}}^{(t)}\left(\mathbf{N}_{i_{k}}^{(t)}\right)\right\},\left\{\mathbf{W}_{i_{k}}^{\text {aux }}\right\}\right) .
\end{aligned}
$$

- Proof of objective function reduction in Step 4: Step 4 consists on updating $\mathbf{W}_{i_{k}}^{(t)}, \forall i, \forall k$. As $f_{o}($.$) is convex on$ $\left\{\mathbf{W}_{i_{k}}\right\}$, we can update them all by following (21) for a set 
of fixed $\left\{\mathbf{T}_{i_{k}}^{(t)}\right\}$ and $\left\{\mathbf{R}_{i_{k}}^{(t)}\right\}$, and thereby a reduction in the objective function in (36) is guaranteed, i.e.

$$
\begin{aligned}
& f_{o}\left(\left\{\mathbf{T}_{i_{k}}^{(t)}\left(\mathbf{\Upsilon}_{k}^{(t)}\right)\right\},\left\{\mathbf{R}_{i_{k}}^{(t)}\left(\mathbf{N}_{i_{k}}^{(t)}\right)\right\},\left\{\mathbf{W}_{i_{k}}^{\text {aux }}\right\}\right) \geq \\
& f_{o}\left(\left\{\mathbf{T}_{i_{k}}^{(t)}\left(\mathbf{\Upsilon}_{k}^{(t)}\right)\right\},\left\{\mathbf{R}_{i_{k}}^{(t)}\left(\mathbf{N}_{i_{k}}^{(t)}\right)\right\},\left\{\mathbf{W}_{i_{k}}^{(t)}\right\}\right) .
\end{aligned}
$$

Therefore, with the proof of a objective function reduction in these 4 steps, monotonic convergence of the algorithm for D-CoP in Table II is demonstrated at each iteration $t$ :

$$
\begin{aligned}
& f_{o}\left(\left\{\mathbf{T}_{i_{k}}^{(t-1)}\right\},\left\{\mathbf{R}_{i_{k}}^{(t-1)}\left(\mathbf{N}_{i_{k}}^{(t-1)}\right)\right\},\left\{\mathbf{W}_{i_{k}}^{(t-1)}\right\}\right) \geq \\
& f_{o}\left(\left\{\mathbf{T}_{i_{k}}^{(t)}\left(\mathbf{\Upsilon}_{k}^{(t)}\right)\right\},\left\{\mathbf{R}_{i_{k}}^{(t)}\left(\mathbf{N}_{i_{k}}^{(t)}\right)\right\},\left\{\mathbf{W}_{i_{k}}^{(t)}\right\}\right) .
\end{aligned}
$$

Steps 3 and 4-1 could be interchanged and convergence would also be guaranteed. However, it is indispensable to update $\left\{\mathbf{N}_{i_{k}}\right\}$ and $\left\{\mathbf{\Upsilon}_{k}\right\}$ in different steps to guarantee convergence.

\section{REFERENCES}

[1] S. Lagen, A. Agustin, and J. Vidal, "Distributed Inter-Cluster Interference Management for CoMP-based Cellular Networks," IEEE Global Communications Conference, pp. 4204-4209, Dec. 2013.

[2] P. Bhat, S. Nagata, L. Campoy, I. Berberana, T. Derham, G. Liu, X. Shen, P. Zong, and J. Yang, "LTE-Advanced: An Operator Perspective," IEEE Communications Magazine, vol. 50, pp. 104-114, Feb. 2012.

[3] N. Bhushan et al., "Network densification: the dominant theme for wireless evolution into 5G," IEEE Communications Magazine, vol. 52, pp. 82-89, Feb. 2014.

[4] 3rd Generation Partnership Project (3GPP), http://www.3gpp.org/.

[5] 3GPP TR 36.912, Feasibility study for Further Advancements for EUTRA (LTE-Advanced), Release 11, v11.0.0, Sep. 2012.

[6] 3GPP TR 36.872, Small cell enhancements for E-UTRA and E-UTRAN - Physical layer aspects, Release 12, v12.1.0, Dec. 2013.

[7] Accelleran, "The essential importance of LTE TDD for small cell deployments," Jul. 2013.

[8] Z.-Q. Luo and S. Zhang, "Dynamic Spectrum Management: Complexity and Duality," IEEE J. Sel. Topics in Signal Processing, vol. 2, pp. 57-53, Feb. 2008

[9] C. Shi, D. A. Schmidt, R. A. Berry, M. L. Honig, and W. Utschick, "Distributed Interference Pricing for the MIMO Interference Channel," in IEEE Int. Conf. on Communications, Jun. 2009.

[10] G. Scutari, D. P. Palomar, F. Facchinei, and J.-S. Pang, "Distributed Dynamic Pricing for MIMO Interfering Multiuser Systems: A Unified Approach," in IEEE Int. Conf. on Network Games, Control and Optimization, Oct. 2011.

[11] Q. Shi, M. Razaviyayn, Z.-Q. Luo, and C. He, "An Iteratively Weighted MMSE Approach to Distributed Sum-Utility Maximization for a MIMO Interfering Broadcast Channel," IEEE Trans. on Signal Processing, vol. 59, pp. 4331-4340, Sep. 2011.

[12] S. Christensen, R. Agarwal, E. Carvalho, and J. M. Cioffi, "Weighted Sum-Rate Maximization using Weighted MMSE for MIMO-BC Beamforming Design," IEEE Trans. on Wireless Commun., vol. 7, pp. 47924799, Dec. 2008.

[13] H. Hassibi and B. M. Hochwald, "How much training is needed in multiple-antenna wireless links?," IEEE Trans. on Information Theory, vol. 49, pp. 951-963, Apr. 2003.

[14] M. Biguesh and A. Gershman, "Training-Based MIMO Channel Estimation: A Study of Estimator Tradeoffs and Optimal Training Signals," IEEE Trans. on Signal Processing, vol. 54, pp. 884-893, Mar. 2006.

[15] X. Zhang, D. P. Palomar, and B. Ottersten, "Statistically Robust Design of Linear MIMO Transceivers," IEEE Trans. on Signal Processing, vol. 56, pp. 3678-3689, Aug. 2008.

[16] D. P. Bertsekas, Nonlinear Programming. 2nd ed. Belmong, MA: Athena Scientific, 1999.

[17] S. Boyd and L. Vandenbergue, Convex optimization. Cambridge, 2004.

[18] 3GPP TR 36.829, Enhanced performance requirement for LTE User Equipment (UE), Release 11, v11.1.0, Jan. 2013.

[19] G. Scutari, F. Facchinei, P. Song, D. P. Palomar, and J.-S. Pang, "Decomposition by Partial Linearization: Parallel Optimization of MultiAgent Systems," IEEE Trans. on Signal Processing, vol. 62, pp. 641656, Feb. 2014

[20] 3GPP TR 36.814, Further advancements for E-UTRA physical layer aspects, Release 9, v9.0.0, Mar. 2010.
[21] S. Lagen, A. Agustin, and J. Vidal, "Decentralized Beamforming with Coordinated Sounding for Inter-Cell Interference Management," European Wireless Conference, pp. 111-116, May 2014.

[22] 3GPP R1-092359, Huawei, Hardware calibration requirement for dual layer beamforming, Jul. 2009.

[23] 3GPP R1-091368, Nokia Siemens Networks, Nokia, Performance comparison between Tx diversity and single stream precoding, Mar. 2009.

[24] F. Huang, Y. Wang, J. Geng, and D. Yang, "Antenna mismatch and calibration problem in coordinated multi-point transmission system," IET Communications, vol. 6, pp. 289-299, Feb. 2012.

[25] 3GPP R1-134291, DAC-UPC, IAESI, Coordinated Sounding for CoMP BF (CoMP BF-CoS) exploiting TDD channel reciprocity, Oct. 2013.

[26] G. Scutari, D. P. Palomar, and S. Barbarossa, "The MIMO Iterative Waterfilling Algorithm," IEEE Trans. on Signal Processing, vol. 57, pp. 1917-53, May. 2009.

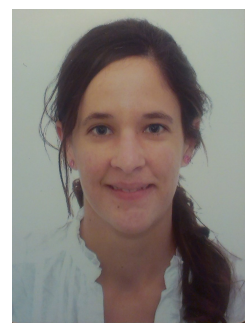

Sandra Lagen was born in Barcelona, Spain, in 1988. She received the M.S. degree in Telecommunications Engineering from Universitat Politècnica de Catalunya (UPC), Barcelona, Spain, in 2011, and since 2013 she is working towards her Ph.D. degree within the department of Signal Theory and Communications at UPC. From 2010 to 2011, she was with Abertis Telecom, Barcelona. In 2012 she joined the department of Signal Theory and Communications at UPC as a research assistant, where she has participated in the EC funded projects FREEDOM and TROPIC. Her research interests are focused on communication theory and signal processing for interference management in cellular networks.

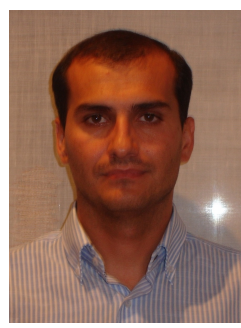

Adrian Agustin received the M.S. degrees in Telecommunication Engineering and Electronic Engineering and the $\mathrm{Ph} . \mathrm{D}$. degree from Universitat Politècnica de Catalunya (UPC), Barcelona, Spain, in 2000, 2002 and 2008, respectively. From 2000 to 2002 , he was with Indra-Espacio, Barcelona, working on research and development of code synchronization techniques for DS-CDMA. In 2002 he joined the Signal Theory and Communications department at UPC as a research assistant. In 2008, he became a research associate. He has participated in the EC funded projects SATURN, ROMANTIK, FIREWORKS, ROCKET, FREEDOM, TROPIC and TUCAN3G. His research interests include wireless multi-user MIMO, interference management and interference alignment.

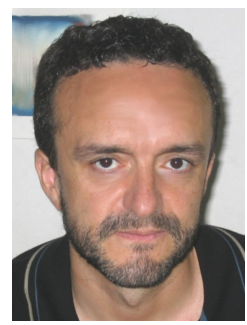

Josep Vidal received the Telecommunication Engineering and the $\mathrm{Ph}$. D. degrees from the Universitat Politècnica de Catalunya (UPC), Barcelona, where he is Professor at the Signal Theory and Communications department. From 1989 to 1990 he joined the LTS at the Ecole Polytechnique de Lausanne as associate researcher. He was awarded UPC Premio Extraordinario de Doctorado in 1996. His research interests are in statistical signal processing, information and communication theory, areas in which he has authored more than 150 journal and conference papers. Since 2002 has coordinated collaborative EC-funded projects ROMANTIK, FIREWORKS, ROCKET, FREEDOM, TROPIC and TUCAN3G, belonging to the FP5, FP6 and FP7 programmes, all in different areas of MIMO relay communications, self-organization, cooperative transmission and heterogeneous networks. He has held research appointments with INP Toulouse and University of Hawaii, and has organized several international workshops. From 2011 through 2014 he served as associate editor of IEEE Transactions on Signal Processing. 